CERN-TH.6337/91

IFUM 413/FT

hepth@xxx/9201067

\title{
EXACT S-MATRICES FOR NONSIMPLY-LACED AFFINE TODA THEORIES
}

\author{
G.W. DELIUS and M.T. GRISARU \\ Theory Division, CERN, 1211 Geneva 23, Switzerland \\ and \\ D. ZANON \\ Dipartimento di Fisica dell' Università di Milano and \\ INFN, Sezione di Milano, I-20133 Milano, Italy
}

\begin{abstract}
We derive exact, factorized, purely elastic scattering matrices for the affine Toda theories based on the nonsimply-laced Lie algebras $a_{2 n-1}^{(2)}, b_{n}^{(1)}$, $c_{n}^{(1)}, d_{n}^{(2)}$, and $g_{2}^{(1)}$, as well as the superalgebras $A^{(4)}(0,2 n)$ and $B^{(1)}(0, n)$.
\end{abstract}

CERN-TH.6337/91

IFUM 413/FT

December 1991

${ }^{*}$ On leave from Brandeis University, Waltham, MA 02254, USA

Work partially supported by the National Science Foundation under grant PHY-88-18853 and by INFN. 


\section{Introduction}

A few years ago, in the course of investigating perturbations of conformal field theories, Zamolodchikov considered a class of integrable perturbations that lead to theories with massive excitations whose natural description is in terms of their S-matrices [1]. This resulted in a renewal of interest in S-matrices for integrable two-dimensional systems and, because of connections of the original work to the Lie algebra $E_{8}$ on one hand, and to Toda theories on the other hand, a flurry of activity developed around the construction of S-matrices for affine Toda theories based on various Lie algebras. This construction was successfully carried out for the case of affine Toda theories based on the simply-laced algebras $a_{n}^{(1)}, d_{n}^{(1)}$ and $e_{6,7,8}^{(1)}$, but, except for $a_{2 n}^{(2)}$, failed utterly for the families of nonsimply-laced algebras $a_{2 n-1}^{(2)}, b_{n}^{(1)}$, $c_{n}^{(1)}, d_{n+1}^{(2)}$ as well as for $d_{4}^{(3)}, e_{6}^{(2)}, f_{4}^{(1)}$ and $g_{2}^{(1)}$ [2, 3, 4. 4 . Furthermore, extending the construction to the case of affine Toda theories based on those Lie superalgebras which have massive excitations only, we were able to construct S-matrices for the Lie superalgebras $A^{(2)}(0,2 n-1)$ and $C^{(2)}(n+1)$ (whose bosonic root subsystem is simply-laced), but not for $B^{(1)}(0, n)$ and $A^{(4)}(0,2 n)$ [5]. In this paper we remedy this situation.

Affine Toda theories based on Lie algebras are massive two-dimensional bosonic field theories represented by lagrangians of the form

$$
\mathcal{L}=-\frac{1}{2 \beta^{2}} \vec{\phi} \square \vec{\phi}-\frac{\mu^{2}}{\beta^{2}} \sum_{i} q_{i} e^{\vec{\alpha}_{i} \cdot \vec{\phi}}
$$

where the $\vec{\alpha}_{i}$ are the simple roots of a rank $n$ Lie algebra augmented by (the negative of) a maximal root and $\vec{\phi}=\left(\phi_{1}, \phi_{2}, \ldots \phi_{n}\right)$ are bosonic fields describing $n$ massive particles. For the simply-laced theories all the roots have the same length. (For the case of Lie superalgebras the set of roots is divided into bosonic and fermionic ones, and the lagrangians contain in general fermions as well.) The Kač labels $q_{i}$ are such that $\sum q_{i} \vec{\alpha}_{i}=0$ and the Kač label corresponding to the maximal root is normalized to unity. We define $h=\sum_{i} q_{i}$ and refer to it, somewhat imprecisely, as the Coxeter number. The coupling constant is denoted by $\beta$ and $\mu$ sets the mass scale. For notational convenience we will often omit these two constants.

The Toda field equations may be viewed as integrability conditions for a Lax pair. From this follows the existence of an infinite set of currents $J_{ \pm}^{(s)}$ of increasing spin $s$ (the first of which is the stress tensor) which are conserved by virtue of the field equations. Thus, these theories are classically integrable: the lagrangian above admits an infinite number of symmetries described by the corresponding conserved charges. At the quantum level, the existence of such symmetries has a profound effect on the structure of the scattering amplitudes of these theories: the $n$-particle S-matrices factorize into a product of elastic two-particle S-matrices satisfying YangBaxter relations. In principle, some additional assumptions of analyticity, unitarity and bootstrap principle [6] should allow them to be determined exactly.

Elastic, unitary S-matrices for a process $a+b \rightarrow a+b$ can be written as products 
of ratios of hyperbolic sines

$$
S_{a b}(\theta)=\prod(x), \quad(x) \equiv \frac{\operatorname{sh}\left(\frac{\theta}{2}+\frac{i \pi}{2 h} x\right)}{\operatorname{sh}\left(\frac{\theta}{2}-\frac{i \pi}{2 h} x\right)}
$$

where $\theta=\theta_{a}-\theta_{b}$ is the relative rapidity and $h$ can be identified with the Coxeter number of the Lie algebra. For $x<h \bmod 2 h$ these S-matrices have physical sheet simple poles at $\theta=\frac{i \pi x}{h}$ and these can be interpreted as elementary particle poles from s-channel or u-channel exchange, with masses related to the values of $x$. These values are constrained by the bootstrap principle: any physical sheet simple pole must be interpretable in this manner and the corresponding particle must appear in the set $\{a, b, \ldots\}$ which labels the S-matrix. Values of $x$ outside the above range are not restricted. Coupling constant dependence (with $S(\beta=0)=1$ ) may be introduced by means of additional blocks $(x \pm B)^{-1}$. Here $B(\beta)$ (with $B(0)=0$ ) is a priori arbitrary, but there is good evidence that $B=\frac{\beta^{2}}{2 \pi}\left(1+\frac{\beta^{2}}{4 \pi}\right)^{-1}$. Thus, typically

$$
S_{a b}(\theta)=\prod_{x \in A_{a b}} \frac{(x)}{(x \pm B)}
$$

for some set $A_{a b}$. If the particles are self-conjugate, crossing symmetry $S(\theta)=$ $S(i \pi-\theta)$ implies that along with the blocks $(x)(x-B)^{-1}$ the above product also contains the blocks $(h-x)(h-x+B)^{-1}$ The blocks in the numerator determine pole positions, while the blocks in the denominator determine coupling constant dependence.

Additional restrictions come from the assumption of the S-matrix bootstrap which says that if $S_{a b}$ has a pole at $\theta=\theta_{a b}^{c}$ corresponding to the s-channel exchange of a particle $c$, then

$$
S_{c d}(\theta)=S_{a d}\left(\theta+\bar{\theta}_{a c}^{b}\right) S_{b d}\left(\theta-\bar{\theta}_{b c}^{a}\right)
$$

where $\bar{\theta}=i \pi-\theta$. The bootstrap principle and the S-matrix bootstrap severely restrict the choice of s-channel exchanges. In the usual construction one requires eq. (1.4) to be satisfied by the numerator of eq.(1.3) independently of the denominator and this reduces the set $A_{a b}$ to integers, fixing the mass ratios of the elementary particles to values independent of the coupling constant.

For simply-laced Toda theories, the S-matrices are constructed by first requiring tree-level consistency with the classical mass spectra and with the three-point couplings (as extracted from the Toda lagrangians) which determine possible oneparticle exchanges. For example, for the $a_{n}^{(1)}$ affine Toda theory, with Coxeter number $h=n+1$ and classical mass spectrum

$$
m_{a}=2 \mu \sin \frac{a \pi}{h} \quad, \quad a=1,2, \ldots, n
$$

the proposed S-matrix is given by [2]

$$
S_{a b}=\{a+b-1\}\{a+b-3\} \ldots\{|a-b|+1\}
$$

in terms of the convenient notation [3]

$$
\{x\}=\frac{(x+1)(x-1)}{(x+1-B)(x-1+B)}
$$


At the quantum level one checks that (one-loop) corrections do not affect the mass spectrum (aside from an overall rescaling that can be absorbed into the mass scale). Other important checks consist in correctly locating and identifying various higherorder poles as anomalous threshold singularities [3]. Similar constructions exist for the other simply-laced cases and for two theories based on Lie superalgebras.

However, such construction has failed for affine Toda theories based on nonsimplylaced algebras on several counts: a) As mentioned above, the bootstrap principle leads to restrictions on the masses of the particles. These restrictions are consistent with the classical masses, for both simply-laced and nonsimply-laced theories, but for the nonsimply-laced theories radiative corrections distort the mass spectrum in a manner that at first sight seems incompatible with bootstrap restrictions [3, 4, [7]. b) For the nonsimply-laced Toda theories an explanation of higher-order poles as anomalous thresholds has failed to account for all the singularities of proposed Smatrices. c) As discussed in Refs. [8, 9] certain anomalous threshold singularities lead to a breakdown of the charge conservation rules used in, and implied by, the bootstrap procedure. These facts have led to speculations that factorizable, elastic S-matrices do not exist for these Toda theories, perhaps because the classical integrability breaks down at the quantum level due to anomalies.

In Ref. [9] we have demonstrated that at the quantum level, Toda theories for both simply-laced and nonsimply-laced algebras do have higher spin conserved currents, albeit modified by quantum corrections. Therefore, the difficulties encountered in attempts to construct elastic, factorizable S-matrices for the nonsimplylaced cases cannot be blamed on a failure of the charge conservation laws. What is required is some modification of the usual construction which takes into account the distortion of the mass spectrum, the anomalous threshold structure, and, where it occurs, the breakdown of the bootstrap. As we shall demonstrate, one only has to give up the idea that the blocks $(x)$ which determine particle pole positions satisfy the bootstrap independently of the blocks $(x \pm B)$. This allows $(x)$ to be coupling constant dependent and makes possible the construction of satisfactory S-matrices.

Coupling constant dependence in the numerator blocks of eq.(1.3) should not come as a surprise since quantum corrections do change the mass spectrum. What is remarkable is the fact that the procedure we will describe can be implemented consistently in just a few ways, and that the various nonsimply-laced Toda theories take advantage of this. Thus we are able to construct exact factorized S-matrices for all the families of nonsimply-laced Toda theories $a_{2 n-1}^{(2)}, b_{n}^{(1)}, c_{n}^{(1)}, d_{n}^{(2)}$, and the $g_{2}^{(1)}$ theory, as well as the superalgebra cases $A^{(4)}(0,2 n)$ and $B^{(1)}(0, n)$. We leave the theories based on $d_{4}^{(3)}, e_{6}^{(2)}$ and $f_{4}^{(1)}$ as an exercise for the reader. As we will see, when the dust settles, these S-matrices differ from previous ones in only one respect: the Coxeter number which appears in the mass formulas and in the S-matrix blocks has to be replaced by a "renormalized", coupling constant dependent Coxeter number.

In the next sections we present the construction and the perturbative verifications. For general information about the techniques used the reader should consult some of the literature in the references, in particular [3, 5, 10]. We will proceed in alphabetical order, from $a_{2 n-1}^{(2)}$, to $b_{n}^{(1)}$, to $c_{n}^{(1)}$, etc., giving full details for the first 
nonsimply-laced Toda theory and less for the others, leaving some of the checks of the later theories for the enterprising reader. A brief account of the procedure was presented in Ref. [11].

\section{The S-matrix of the $a_{2 n-1}^{(2)}$ theory}

The Toda theory based on the nonsimply-laced twisted affine Lie algebras $a_{2 n-1}^{(2)}$ is described by the classical lagrangian

$$
\begin{aligned}
\beta^{2} \mathcal{L} & =-\frac{1}{2} \sum_{1}^{n-1} \phi_{a} \square \phi_{a}-\frac{1}{2} \phi_{n} \square \phi_{n} \\
& -2 \sum_{k=2}^{n-1} \exp \left(-\sqrt{\frac{2}{h}} \sum_{1}^{n-1} m_{a} \cos \frac{(2 k-1) a \pi}{h} \phi_{a}\right)-\exp \left(-\sqrt{\frac{2}{h}} \sum_{1}^{n-1}(-1)^{a} m_{a} \phi_{a}\right) \\
& -\exp \left(-\sqrt{\frac{2}{h}} \sum_{1}^{n-1} m_{a} \cos \frac{a \pi}{h} \phi_{a}+\phi_{n}\right)-\exp \left(-\sqrt{\frac{2}{h}} \sum_{1}^{n-1} m_{a} \cos \frac{a \pi}{h} \phi_{a}-\phi_{n}\right)
\end{aligned}
$$

with masses and three-point couplings given by

$$
\begin{array}{cc}
m_{n}^{2}=2, \quad m_{a}^{2}=4 m_{n}^{2} \sin ^{2}\left(\frac{a \pi}{h}\right) \quad a=1, \ldots, n-1, \\
c_{a b c}=-\frac{\beta}{\sqrt{2 h}} m_{a} m_{b} m_{c}=-\frac{4 \beta}{\sqrt{h}} \Delta_{a b c} & \text { if } a+b+c=h \\
c_{a b c}=\frac{\beta}{\sqrt{2 h}} m_{a} m_{b} m_{c}=\frac{4 \beta}{\sqrt{h}} \Delta_{a b c} & \text { if } a \pm b \pm c=0 \\
c_{a n n}=\beta \sqrt{\frac{2}{h}} m_{n}^{2} m_{a} \cos \frac{a \pi}{h}=\frac{4 \beta}{\sqrt{h}} \Delta_{a n n} &
\end{array}
$$

All other three-point couplings are zero. Here $\Delta_{a b c}$ represents the area of a triangle with sides $m_{a}, m_{b}, m_{c}$. We have introduced the Coxeter number $h=2 n-1$ and set the overall mass-scale $\mu=1$.

In perturbation theory the only divergences one encounters in these bosonic systems come from tadpoles. A finite quantum theory can be defined by absorbing such divergences into a renormalization of the mass scale and, for these nonsimplylaced cases, a shift in the vacuum expectation values of the fields which leads to a renormalization of the Kač labels. The bare Kač labels and the bare mass-scale are chosen such that the quantum lagrangian is identical in form to the classical lagrangian in eq.(2.1), but with normal-ordered exponentials. As we will mention later on, a slightly different prescription is required when fermions are present.

At the one-loop level radiative corrections lead to mass shifts that have been computed in Ref. [7]. They give

$$
\delta \frac{m_{a}^{2}}{m_{n}^{2}}=-\frac{a \beta^{2}}{h^{2}} \sin \frac{2 a \pi}{h} .
$$


(In contrast, for a simply-laced theory and also, exceptionally, for the $a_{2 n}^{(2)}$ theory the mass ratios do not receive corrections.) Without loss of generality we will write the radiatively-corrected masses, with reference to eq.(2.2), as

$$
\widetilde{m}_{a}^{2}=4 \widetilde{m}_{n}^{2} \sin ^{2}\left(\frac{\pi}{h}\left(a+\epsilon_{a}(\beta)\right)\right)
$$

At this stage, the $\epsilon_{a}(\beta)$ are in principle computable to any order of perturbation theory. The S-matrix will be defined with respect to these masses.

In Ref. [9] we have demonstrated the existence of a quantum-conserved spin 3 current for the $n=2$ case,

$$
\begin{aligned}
J_{+}^{(3)}= & \left(1+\frac{\alpha}{2}\right)\left(\partial_{+} \phi_{1}\right)^{2}\left(\partial_{+} \phi_{2}\right)^{2}-\frac{\alpha}{12}\left(1+3 \alpha+\alpha^{2}\right)\left(\partial_{+}^{2} \phi_{1}\right)^{2}-\frac{\alpha}{12}\left(\partial_{+} \phi_{1}\right)^{4}-\frac{\alpha}{12}\left(\partial_{+} \phi_{2}\right)^{4} \\
& +\left(1+\frac{23}{12} \alpha+\alpha^{2}+\frac{1}{6} \alpha^{3}\right)\left(\partial_{+}^{2} \phi_{2}\right)^{2}+\left(2+3 \alpha+\alpha^{2}\right) \partial_{+} \phi_{1} \partial_{+} \phi_{2} \partial_{+}^{2} \phi_{2}
\end{aligned}
$$

(with a suitable expression for $J_{-}^{(3)}$ ). Here $\alpha=\frac{\beta^{2}}{2 \pi}$. We expect that a similar conserved current exists for all the $a_{2 n-1}^{(2)}$ Toda theories. Together with the renormalized stress tensor this defines corresponding spin 1 and spin 3 charges

$$
Q^{(1)}=\int d x^{+} T_{++} \quad, \quad Q^{(3)}=\int d x^{+} J_{+}^{(3)}
$$

whose presence guarantees the existence of factorizable elastic S-matrices. The bootstrap procedure should then determine them. However, as described in the above reference, the presence of certain anomalous threshold singularities implies a breakdown of the usual bootstrap results when the $\left\langle\phi_{a} \phi_{b} \phi_{h-a-b}\right\rangle$ vertex is involved. We will see later on precisely where and how this occurs.

\subsection{The S-matrix}

We construct the S-matrix by following procedures similar to those used in the simply-laced case, but with two important differences: we admit that the S-matrix has simple particle poles at positions shifted away from the classical mass values by radiative corrections, and we relax the bootstrap principle since some simple poles are shifted away from their single-particle positions due to anomalous threshold effects. (We will prove that all the shifted poles we find can be accounted for in this fashion.)

Assuming that higher-spin quantum-conserved currents similar to those in eq.(2.6) exist for all the $a_{2 n-1}^{(2)}$ theories, we postulate the existence of purely elastic two-body amplitudes. We start by determining the element $S_{n n}$. Since the three-point couplings in eq.(2.3) suggest that all the particles $\phi_{1} \cdots \phi_{n-1}$ appear as intermediate particles in this process the S-matrix bootstrap will allow the determination of all the other amplitudes. The intermediate particles lead to both $s$ - and $u$-channel poles in $S_{n n}$. In the rapidity plane these poles are located at:

$$
\begin{array}{lll}
s-\widetilde{m}_{a}^{2}=2 \widetilde{m}_{n}^{2}(1+\operatorname{ch} \theta)-\widetilde{m}_{a}^{2}=0 & \Rightarrow & \theta=\frac{i \pi}{h}\left(h-2 a-2 \epsilon_{a}\right) \\
u-\widetilde{m}_{a}^{2}=2 \widetilde{m}_{n}^{2}(1-\operatorname{ch} \theta)-\widetilde{m}_{a}^{2}=0 & \Rightarrow & \theta=\frac{i \pi}{h}\left(2 a+2 \epsilon_{a}\right)
\end{array}
$$


We must reproduce these poles with the building blocks in eq.(1.2]). The choice

$$
S_{n n}^{(\min )}=\prod_{a=1}^{n-1}\left(2 a+2 \epsilon_{a}\right)\left(h-2 a-2 \epsilon_{a}\right)
$$

has the right pole structure. In order for the S-matrix to reduce to the identity matrix when the coupling constant $\beta$ is zero we make the crossing symmetric Ansatz

$$
S_{n n}=\prod_{a=0}^{n-1} \frac{\left(2 a+2 \epsilon_{a}\right)\left(h-2 a-2 \epsilon_{a}\right)}{\left(2 a+2 \eta_{a}\right)\left(h-2 a-2 \eta_{a}\right)}
$$

where $\epsilon_{a}$ and $\eta_{a}$ are both zero when $\beta=0$. We let the product start at $a=0$. This is required for agreement with the tree level amplitudes. None of the extra blocks which we introduced in eq.(2.10) should produce additional poles on the physical sheet (i.e., for $0<\theta<i \pi$ ) and this requires

$$
\frac{h}{2}-a \geq \eta_{a} \geq-a \quad, \quad \epsilon_{0} \leq 0
$$

The bootstrap principle allows the determination of the S-matrix elements $S_{a n}$, $a=1 \cdots n-1$, through

$$
S_{a n}(\theta)=S_{n n}\left(\theta+\frac{1}{2} \theta_{n n}^{a}\right) S_{n n}\left(\theta-\frac{1}{2} \theta_{n n}^{a}\right)
$$

Here $\theta_{n n}^{a}$ is the relative rapidity at which $S_{n n}$ has the s-channel pole corresponding to particle $a$, i.e., $\theta_{n n}^{a}=\frac{i \pi}{h}\left(h-2 a-2 \epsilon_{a}\right)$, cf. eq.(2.8). Using

$$
(x)_{\left(\theta+\frac{i \pi}{h} \rho\right)} \cdot(x)_{\left(\theta-\frac{i \pi}{h} \rho\right)}=(x+\rho)_{(\theta)} \cdot(x-\rho)_{(\theta)}
$$

we find

$$
\begin{aligned}
S_{a n}= & \prod_{p=0}^{n-1} \frac{\left(2 p+2 \epsilon_{p}-\frac{h}{2}+a+\epsilon_{a}\right)\left(h-2 p-2 \epsilon_{p}-\frac{h}{2}+a+\epsilon_{a}\right)}{\left(2 p+2 \eta_{p}-\frac{h}{2}+a+\epsilon_{a}\right)\left(h-2 p-2 \eta_{p}-\frac{h}{2}+a+\epsilon_{a}\right)} \\
& \times \frac{\left(2 p+2 \epsilon_{p}+\frac{h}{2}-a-\epsilon_{a}\right)\left(h-2 p-2 \epsilon_{p}+\frac{h}{2}-a-\epsilon_{a}\right)}{\left(2 p+2 \eta_{p}+\frac{h}{2}-a-\epsilon_{a}\right)\left(h-2 p-2 \eta_{p}+\frac{h}{2}-a-\epsilon_{a}\right)}
\end{aligned}
$$

This expression has a large number of poles, many more than perturbation theory can be expected to explain. However for special values of $\epsilon_{a}$ and $\eta_{a}$ many of the blocks cancel each other, as we now show. We rewrite the expression in eq.(2.14) by using the relations $(x \pm 2 h)=(x)$ and $(-x)=(x)^{-1}$ and bring some of the blocks involving $\eta$ to the numerator. We write

$$
S_{a n}(\theta)=\frac{N(\epsilon, \eta)}{D(\epsilon, \eta)}
$$


where

$$
\begin{aligned}
N(\epsilon, \eta)= & \prod_{p=0}^{n-1}\left(\frac{h}{2}+2 p-a+2 \epsilon_{p}-\epsilon_{a}\right)\left(\frac{h}{2}-2 p+a-2 \epsilon_{p}+\epsilon_{a}\right) \\
\cdot & \left(\frac{h}{2}-2 p-a-2 \eta_{p}-\epsilon_{a}\right)\left(\frac{h}{2}+2 p+a+2 \eta_{p}+\epsilon_{a}\right) \\
= & \prod_{p=n-a}^{n-1}\left(\frac{h}{2}-a-2 p-2 \eta_{p}-\epsilon_{a}\right) \prod_{p=n-a}^{n-1}\left(\frac{h}{2}+a+2 p+2 \eta_{p}+\epsilon_{a}\right) \\
& \prod_{p=0}^{n-a-1}\left(\frac{h}{2}-a-2 p-2 \eta_{p}-\epsilon_{a}\right) \prod_{p=a}^{n-1}\left(\frac{h}{2}+a-2 p-2 \epsilon_{p}+\epsilon_{a}\right) \\
& \prod_{p=1}^{a}\left(\frac{h}{2}-a+2 p+2 \epsilon_{p}-\epsilon_{a}\right) \prod_{p=0}^{a-1}\left(\frac{h}{2}+a-2 p-2 \epsilon_{p}+\epsilon_{a}\right) \\
& \prod_{p=a+1}^{n-1}\left(\frac{h}{2}-a+2 p+2 \epsilon_{p}-\epsilon_{a}\right) \prod_{p=1}^{n-a-1}\left(\frac{h}{2}+a+2 p+2 \eta_{p}+\epsilon_{a}\right) \\
& \cdot\left(\frac{h}{2}-a+2 \epsilon_{0}-\epsilon_{a}\right)\left(\frac{h}{2}+a+2 \eta_{0}+\epsilon_{a}\right)
\end{aligned}
$$

and $D(\epsilon, \eta)$ is obtained by the interchange $2 \epsilon_{p} \leftrightarrow 2 \eta_{p}$ in the above expression. After making suitable changes of the variable $p$ in each of the above products we can rewrite

$$
\begin{aligned}
N(\epsilon, \eta)= & \prod_{p=n-a}^{n-1}\left(\frac{h}{2}-a-2 p-2 \eta_{p}-\epsilon_{a}\right)\left(\frac{h}{2}-a-2 p+2 \eta_{h-a-p}+\epsilon_{a}\right) \\
& \prod_{p=1}^{a-1}\left(\frac{h}{2}-a-2 p-2 \eta_{p}-\epsilon_{a}\right)\left(\frac{h}{2}-a-2 p-2 \epsilon_{a+p}+\epsilon_{a}\right) \\
& \prod_{p=1}^{a-1}\left(\frac{h}{2}-a+2 p+2 \epsilon_{p}-\epsilon_{a}\right)\left(\frac{h}{2}-a+2 p-2 \epsilon_{a-p}+\epsilon_{a}\right) \\
& \prod_{p=a+1}^{n-1}\left(\frac{h}{2}-a+2 p+2 \epsilon_{p}-\epsilon_{a}\right)\left(\frac{h}{2}-a+2 p+2 \eta_{p-a}+\epsilon_{a}\right) \\
& \cdot\left(\frac{h}{2}-a+2 \epsilon_{0}-\epsilon_{a}\right)\left(\frac{h}{2}+a+2 \eta_{0}+\epsilon_{a}\right)\left(\frac{h}{2}-a-2 \eta_{0}-\epsilon_{a}\right) \\
& \cdot\left(\frac{h}{2}-a-\epsilon_{a}\right)\left(\frac{h}{2}+a+\epsilon_{a}\right)\left(\frac{h}{2}+a-2 \epsilon_{0}+\epsilon_{a}\right)
\end{aligned}
$$


with a corresponding expression for $D(\epsilon, \eta)$ :

$$
\begin{aligned}
D(\epsilon, \eta)= & \prod_{p=n-a}^{n-1}\left(\frac{h}{2}-a-2 p-2 \epsilon_{p}-\epsilon_{a}\right)\left(\frac{h}{2}-a-2 p+2 \epsilon_{h-a-p}+\epsilon_{a}\right) \\
& \prod_{p=1}^{a-1}\left(\frac{h}{2}-a-2 p-2 \epsilon_{p}-\epsilon_{a}\right)\left(\frac{h}{2}-a-2 p-2 \eta_{a+p}+\epsilon_{a}\right) \\
& \prod_{p=1}^{a-1}\left(\frac{h}{2}-a+2 p+2 \eta_{p}-\epsilon_{a}\right)\left(\frac{h}{2}-a+2 p-2 \eta_{a-p}+\epsilon_{a}\right) \\
& \prod_{p=a+1}^{n-1}\left(\frac{h}{2}-a+2 p+2 \eta_{p}-\epsilon_{a}\right)\left(\frac{h}{2}-a+2 p+2 \epsilon_{p-a}+\epsilon_{a}\right) \\
& \cdot\left(\frac{h}{2}-a+2 \eta_{0}-\epsilon_{a}\right)\left(\frac{h}{2}+a+2 \epsilon_{0}+\epsilon_{a}\right)\left(\frac{h}{2}-a-2 \epsilon_{0}-\epsilon_{a}\right) \\
& \cdot\left(\frac{h}{2}-a-2 \eta_{a}+\epsilon_{a}\right)\left(\frac{h}{2}+a+2 \eta_{a}-\epsilon_{a}\right)\left(\frac{h}{2}+a-2 \eta_{0}+\epsilon_{a}\right)
\end{aligned}
$$

We observe that one choice which cancels many poles is $\epsilon_{a}=0$ and $\eta_{a}=\frac{B}{2}$ for all $a$ and some $B=B(\beta)$. Then we are left with

$$
S_{a n}^{(\text {unren })}=\prod_{p=1}^{2 a-1} \frac{\left(\frac{h}{2}-a+p\right)^{2}}{\left(\frac{h}{2}-a+p+B\right)\left(\frac{h}{2}-a+p-B\right)} \frac{\left(\frac{h}{2}-a\right)}{\left(\frac{h}{2}-a+B\right)} \frac{\left(\frac{h}{2}+a\right)}{\left(\frac{h}{2}+a-B\right)}
$$

This choice corresponds to unrenormalized mass ratios and is not relevant here.

However there is a choice which has even fewer poles: we require

$$
\begin{aligned}
-2 \eta_{p}-\epsilon_{a}=2 \epsilon_{h-a-p}+\epsilon_{a} & \rightarrow \epsilon_{h-a-p}=-\epsilon_{a}-\eta_{p} \\
2 \eta_{h-a-p}+\epsilon_{a}=-2 \epsilon_{p}-\epsilon_{a} & \rightarrow \eta_{h-a-p}=-\epsilon_{a}-\epsilon_{p}
\end{aligned}
$$

for $p=n-a, \ldots, n-1$, which entirely cancels the first line in $N(\epsilon, \eta)$ against that in $D(\epsilon, \eta)$. Similarly, requiring

$$
\begin{aligned}
-2 \eta_{p}-\epsilon_{a}=-2 \eta_{a+p}+\epsilon_{a} & \rightarrow \eta_{a+p}=\epsilon_{a}+\eta_{p} \\
-2 \epsilon_{a+p}+\epsilon_{a}=-2 \epsilon_{p}-\epsilon_{a} & \rightarrow \epsilon_{a+p}=\epsilon_{a}+\epsilon_{p} \\
2 \epsilon_{p}-\epsilon_{a}=2 \epsilon_{p-a}+\epsilon_{a} & \rightarrow \epsilon_{p-a}=\epsilon_{p}-\epsilon_{a} \\
2 \eta_{p}-\epsilon_{a}=2 \eta_{p-a}+\epsilon_{a} & \rightarrow \eta_{p-a}=\eta_{p}-\epsilon_{a}
\end{aligned}
$$

cancels the second and fourth lines. The above equations have the general solution

$$
\epsilon_{a}=a \epsilon \quad, \quad \eta_{a}=(a-h) \epsilon \quad a=1,2, \cdots, n-1
$$

for some arbitrary $\epsilon(\beta)$ with $\epsilon(0)=0$. Additional poles from the last two lines in eq. 2.17) may be cancelled by choosing

$$
\epsilon_{0}=0
$$


and

$$
\eta_{0}=-h \epsilon \quad \text { or } \quad \eta_{0}=0
$$

However, the second choice for $\eta_{0}$ would completely cancel the $a=0$ block in eq.(2.10) and we would fail to account at the tree level for the part of $S_{n n}$ which is independent of $\theta$ (cf. $B(\theta)$ in eq. (2.5) of Ref. [5]. The theory considered there has the same bosonic tree level amplitude as the present theory). The first choice for $\eta_{0}$ gives the description of the S-matrix for the theory at hand. As we discuss later, the second choice leads eventually to the S-matrix for the Toda theory based on the Lie superalgebra $A^{(4)}(0,2 n)$, where a part independent of $\theta$ is indeed absent. We note that $\epsilon(\beta)$ satisfies $-\frac{1}{2 n} \leq \epsilon \leq 0$ in order to fulfill eq.(2.11).

The S-matrix element $S_{a n}$ becomes

$$
\begin{aligned}
S_{a n}= & \prod_{p=1}^{a-1} \frac{\left(\frac{h}{2}+(-a+2 p)(1+\epsilon)\right)^{2}}{\left(\frac{h}{2}+(-a+2 p)(1+\epsilon)-2 h \epsilon\right)\left(\frac{h}{2}+(-a+2 p)(1+\epsilon)+2 h \epsilon\right)} \\
& \times \quad \frac{\left(\frac{h}{2}-a(1+\epsilon)\right)}{\left(\frac{h}{2}-a(1+\epsilon)-2 h \epsilon\right)} \frac{\left(\frac{h}{2}+a(1+\epsilon)\right)}{\left(\frac{h}{2}+a(1+\epsilon)+2 h \epsilon\right)}
\end{aligned}
$$

This expression still has two simple poles at $\theta=\frac{i \pi}{h}\left(\frac{h}{2}+a(1+\epsilon)\right)$ and at $\theta=$ $\frac{i \pi}{h}\left(\frac{h}{2}-a(1+\epsilon)\right)$ as well as several double poles. We discuss the double poles later on and note here that the simple poles correspond to particle $\phi_{n}$ in the intermediate s- and u-channels. Indeed

$$
s-\widetilde{m}_{n}^{2}=\widetilde{m}_{n}^{2}+\widetilde{m}_{a}^{2}+2 \widetilde{m}_{n} \widetilde{m}_{a} \operatorname{ch} \theta-\widetilde{m}_{n}^{2}=0 \quad \text { at } \theta=\frac{i \pi}{h}\left(\frac{h}{2}+a(1+\epsilon)\right)
$$

and similarly for the u-channel.

It is convenient to introduce some simplifying notation:

$$
\begin{gathered}
B=-2 h \frac{\epsilon}{1+\epsilon} \quad, \quad H=\frac{h}{1+\epsilon}=h+\frac{B}{2} \\
(x)_{H}=\frac{\operatorname{sh}\left(\frac{\theta}{2}+\frac{i \pi}{2 H} x\right)}{\operatorname{sh}\left(\frac{\theta}{2}-\frac{i \pi}{2 H} x\right)} \quad, \quad\{x\}_{H}=\frac{(x-1)_{H}(x+1)_{H}}{(x-1+B)_{H}(x+1-B)_{H}}
\end{gathered}
$$

Therefore eq.(2.10) and eq.(2.25) become

$$
\begin{aligned}
& S_{n n}=\prod_{a=0}^{n-1} \frac{(2 a)_{H}(H-2 a)_{H}}{(2 a+B)_{H}(H-2 a-B)_{H}} \\
& S_{a n}=\prod_{p=1}^{a}\left\{\frac{H}{2}+2 p-a-1\right\}_{H}=S_{n a} .
\end{aligned}
$$

In terms of this notation eq. 2.5) implies that the renormalized masses have the same form as the classical masses, but with $h$ replaced by $H$. 
The remaining S-matrix elements $S_{a b}, a, b=1 \cdots n-1$ are obtained by another application of the bootstrap

$$
\begin{aligned}
S_{a b}(\theta)= & S_{n b}\left(\theta+\frac{1}{2} \theta_{n n}^{a}\right) S_{n b}\left(\theta-\frac{1}{2} \theta_{n n}^{a}\right) \\
& =\prod_{p=1}^{b}\{2 p-b-1+a\}_{H}\{H+2 p-b-1-a\}_{H}
\end{aligned}
$$

This expression is symmetric in $a, b$ as one can verify by using relations such as $\prod_{p=0}^{x}\{2 p-x\}_{H}=1$. Crossing symmetry can be easily checked by a change of variable $p \rightarrow-p+b+1$ in one of the terms.

$S_{a b}$ has four simple poles. For $a>b$, these are located at $\frac{H}{i \pi} \theta=(a-b),(H-a+$ $b),(H-a-b),(a+b)$. From the couplings in the lagrangian one expects that they could be identified with s-channel and u-channel exchanges of the particles $\phi_{(a-b)}$ and $\phi_{(a+b)}$ for $a+b<\frac{h}{2}$ or $\phi_{(h-a-b)}$ for $a+b>\frac{h}{2}$. If the pole at $\theta=\frac{i \pi}{H}(a-b)$ corresponds to a particle in the u-channel then this particle has mass

$$
\widetilde{m}^{2}=\widetilde{m}_{a}^{2}+\widetilde{m}_{b}^{2}-2 \widetilde{m}_{a} \widetilde{m}_{b} \cos \frac{\pi}{H}(a-b)=4 \widetilde{m}_{n}^{2} \sin ^{2} \frac{(a-b) \pi}{H}
$$

and this identifies it as particle $\phi_{(a-b)}$. Similarly the pole at $\theta=\frac{i \pi}{H}(H-a+b)$ is identified as the s-channel pole of the same particle.

The same calculation for the pole at $\theta=\frac{i \pi}{H}(a+b)$ leads to a mass

$$
\widetilde{m}^{2}=\widetilde{m}_{a}^{2}+\widetilde{m}_{b}^{2}+2 \widetilde{m}_{a} \widetilde{m}_{b} \cos \frac{\pi}{H}(a+b)=4 \widetilde{m}_{n}^{2} \sin ^{2} \frac{(a+b) \pi}{H}
$$

If $a+b<\frac{h}{2}$ this is the mass of particle $\phi_{(a+b)}$. But if $a+b>\frac{h}{2}$ the pole does not appear at the expected position which should correspond, according to the couplings in eq.(2.3), to the particle $\phi_{(h-a-b)}$. This particle has a mass

$$
\widetilde{m}_{(h-a-b)}^{2}=4 \widetilde{m}_{n}^{2} \sin ^{2} \frac{(h-a-b) \pi}{H}
$$

which is not equal to that in eq.(2.33) due to the fact that $h \neq H$. As we will explain in subsection 2.4, this displacement of the pole and the attendant breakdown of the bootstrap principle is due to the presence of an anomalous threshold singularity.

Aside from some information about the particle content and the existence of particular three-point couplings, as well as the fact that the masses renormalize in a nontrivial manner, we have used very little information about the specific Toda lagrangian. Some choices were made in the course of the construction of the Smatrix: no extra CDD poles and zeroes in $S_{n n}$, and a minimal number of poles in $S_{a n}$. After that, the solution of the bootstrap was essentially unique. However, it is necessary to check the self-consistency of the result, and to show that the S-matrix indeed describes scattering in the particular Toda theory. Consistency amounts to showing that the bootstrap closes on the set of particles we have introduced (or explaining where and why it fails), and in identifying all the higher-order poles as 
anomalous threshold singularities. Contact with the Toda lagrangian consists in checking tree-level agreement, and agreement at the loop-level for masses, coupling constant dependence, and coefficients of the various poles. In particular we must determine the relation between the only free parameter which the S-matrix contains, namely $B$, and the coupling constant $\beta$ of the Toda lagrangian. We expect, from the experience with the simply-laced cases, that $B=\frac{\beta^{2}}{2 \pi}\left(1+\frac{\beta^{2}}{4 \pi}\right)^{-1}$ and we will verify that this is indeed consistent with our results.

In carrying out the checks, we have a choice between using the classical masses or the physical masses. In fact it is more convenient to make the second choice, since our S-matrix is already expressed in terms of (we believe) the correct masses. In this spirit, we would rewrite the lagrangian in terms of these masses and counterterms, and choose the counterterms to appropriately cancel part of the loop corrections to the self-energy. In principle we could do the same for the coupling constants, but to the order to which we are working this will not be necessary.

To conclude this subsection we note that our S-matrix, as well as the predicted renormalized mass spectrum, have the conventional forms encountered in the simplylaced theories, with the only difference that the Coxeter number $h$ must be replaced everywhere with the renormalized Coxeter number $H$.

\subsection{Consistency of the bootstrap}

We have used the single particle poles in $S_{n n}$ to determine all the other matrix elements by bootstrap. We have also identified some single particle poles in these matrix elements and these too could be used in the bootstrap leading to the following consistency conditions:

$$
\begin{array}{rlr}
S_{n n}(\theta) & =S_{a n}\left(\theta+\bar{\theta}_{a n}^{n}\right) S_{n n}\left(\theta-\bar{\theta}_{n n}^{a}\right) & \\
S_{n b}(\theta) & =S_{a b}\left(\theta+\bar{\theta}_{a n}^{n}\right) S_{n b}\left(\theta-\bar{\theta}_{n n}^{a}\right) & \\
S_{(a-b) n}(\theta) & =S_{a n}\left(\theta+\bar{\theta}_{a(a-b)}^{b}\right) S_{b n}\left(\theta-\bar{\theta}_{b(a-b)}^{a}\right) & \\
S_{(a-b) c}(\theta) & =S_{a c}\left(\theta+\bar{\theta}_{a(a-b)}^{b}\right) S_{b c}\left(\theta-\bar{\theta}_{b(a-b)}^{a}\right) & \\
S_{(a+b) n}(\theta) & =S_{a n}\left(\theta+\bar{\theta}_{a(a+b)}^{b}\right) S_{b n}\left(\theta-\bar{\theta}_{b(a+b)}^{a}\right) & a+b<\frac{h}{2} \\
S_{(a+b) c}(\theta) & =S_{a c}\left(\theta+\bar{\theta}_{a(a+b)}^{b}\right) S_{b c}\left(\theta-\bar{\theta}_{b(a+b)}^{a}\right) & a+b<\frac{h}{2}
\end{array}
$$

where $\bar{\theta}_{a b}^{c}=i \pi-\theta_{a b}^{c}$ and $\theta_{a b}^{c}$ is the location of the single particle s-channel pole due to particle $c$ in $S_{a b}$ :

$$
\bar{\theta}_{a n}^{n}=\frac{i \pi}{H}\left(\frac{H}{2}-a\right), \quad \bar{\theta}_{n n}^{a}=\frac{i \pi}{H} 2 a, \quad \bar{\theta}_{a(a-b)}^{b}=\bar{\theta}_{a(a+b)}^{b}=\frac{i \pi}{H} b .
$$

In writing the bootstrap equations eq.(2.35) we have used the fact that

$$
\theta_{a b}^{c}+\theta_{c a}^{b}+\theta_{b c}^{a}=2 \pi i \quad \text { for } a, b, c=1 \cdots n .
$$

The first equality in eq.(2.35) is a simple consequence of eq.(2.12) and the fact that $S(\theta+\pi i) S(\theta)=1$. Similarly the second equation is a consequence of eq.(2.31), 
the 3 rd of the 5 th and the 4 th of the 6 th. The last two equations are real consistency conditions on the bootstrap. They can be checked to be satisfied.

Notice the restriction to $a+b<\frac{h}{2}$. No bootstrap equations arise from the poles in $S_{a b}$ at $\theta=\frac{i \pi}{H}(a+b)$ and $\theta=\frac{i \pi}{H}(h-a-b)$ if $a+b>\frac{h}{2}$ because these cannot be identified as single particle poles.

\subsection{Perturbative checks and coupling constant dependence}

We observe that the $a_{2 n-1}^{(2)}$ lagrangian has the same form, classical mass spectrum and couplings as the bosonic part of the Toda lagrangian for the Lie superalgebra $A^{(2)}(0,2 n-1)$ and therefore the bosonic tree-level S-matrices are the same. Furthermore, in both theories the exact expressions for $S_{a n}(\theta)$ and $S_{a b}(\theta)$ are identical, except for the replacement of $h$ by $H$. Therefore, since $H=h+O\left(\beta^{2}\right)$, and having checked in our previous work [5] that one obtains the correct result at the tree-level, no further checks are needed for these S-matrix elements. On the other hand $S_{n n}$ is different in the two theories, and a separate check is necessary to show that the expression in eq.(2.29) has the correct tree level value. This is straightforward, and indeed we find agreement provided $B=\frac{\beta^{2}}{2 \pi}+O\left(\beta^{4}\right)$. In this respect, extending the product in eq.(2.10) to include the $a=0$ term was crucial.

One-loop checks allow us to determine the coupling constant dependence to order $\beta^{4}$. This is achieved by computing at the one-loop level from the Toda lagrangian the residue of a suitable S-matrix element at a single particle pole and comparing it to the corresponding residue extracted from the exact S-matrix. We find agreement provided $B=\frac{\beta^{2}}{2 \pi}-\frac{\beta^{4}}{8 \pi^{2}}$, i.e. to this order

$$
B=\frac{1}{2 \pi} \frac{\beta^{2}}{1+\frac{\beta^{2}}{4 \pi}}
$$

which is the standard dependence on the coupling constant that one has found in simply-laced theories [12]. We give details of the calculation in Appendix B.

We observe that our S-matrix predicts a very specific manner in which radiative effects shift the masses of the theory:

$$
\widetilde{m}_{a}^{2}=4 \widetilde{m}_{n}^{2} \sin ^{2}\left(\frac{\pi a}{H}\right)
$$

Using $H=h+\frac{B}{2}=h+\frac{\beta^{2}}{4 \pi}+O\left(\beta^{4}\right)$ this is consistent with the one-loop mass corrections in eq.(2.4), and already provides a nontrivial check of our S-matrix and of the restrictions that follow from the bootstrap. It will be interesting to perform a direct two-loop calculation of the mass shifts and compare with those predicted here.

As a further perturbative check, we have computed for the $a_{3}^{(2)}$ case, up to oneloop order, the spin 3 charges of the two particles of the theory [8, 9], and found agreement with those predicted by the exact S-matrix, see eq.(9.3). Additional checks are obtained when we calculate the coefficients of the anomalous threshold singularities in the next subsection. 


\subsection{Anomalous threshold singularities and the shifted poles}

As we have mentioned, in comparing the exact S-matrix to perturbation theory we have a choice between using radiatively corrected physical masses (and counterterms which would just remove the corresponding contributions from self-energy diagrams), or classical masses. In a strict perturbation theory context the latter choice might be more logical, and we would expand everything in powers of the coupling constant. However, it is more economical to make the former choice, since it avoids having to expand denominators such as $\left(s-\widetilde{m}^{2}\right)^{-1}$. Furthermore, in a pure S-matrix context questions about the consistency of the bootstrap and identification of higher order poles should be possible in terms of the masses of the asymptotic fields. In any event, choosing to work with physical, quantum-corrected masses reveals further miraculous ways in which these Toda theories solve their own problems.

In perturbation theory, using physical masses, (or in a pure analyticity-unitarity S-matrix approach) the amplitude $S_{a b}$ for $a+b>n$ would have not only a simple pole corresponding to the particle $\phi_{h-a-b}$ as shown in Fig. 1.a, but also neighboring poles produced as anomalous threshold singularities from the various diagrams in Fig. 1.b,c,d,e,f. Indeed, using the value of the physical masses it is straightforward to check by means of a dual diagram analysis that the triangle diagrams produce pole singularities located at $\theta=\frac{i \pi}{H}(a+b)$ and the crossed box in Fig. 1.d has a double pole at the same position. Specifically, denoting by $\delta$ the shift of the S-matrix pole from its expected position, i.e.

$$
\delta=4 \widetilde{m}_{n}^{2}\left(\sin ^{2} \frac{\pi}{H}(a+b)-\sin ^{2} \frac{\pi}{H}(h-a-b)\right)
$$

and $\sigma=s-\widetilde{m}_{h-a-b}^{2}$ we have the contributions from the six diagrams

$$
\begin{aligned}
(a): & \frac{1}{\sigma} c_{a b(h-a-b)}^{2} \\
(b): & \frac{T_{a b}}{\sigma-\delta} c_{a n n} c_{b n n} c_{a b n n} \\
(c): & \frac{T_{a b}}{\sigma(\sigma-\delta)} c_{a n n} c_{b n n} c_{a b(h-a-b)} c_{n n(h-a-b)} \\
(d): & \frac{R_{a b}}{(\sigma-\delta)^{2}} c_{a n n}^{2} c_{b n n}^{2} \\
(e): & \frac{T_{a b}^{2}}{(\sigma-\delta)^{2}} c_{a n n}^{2} c_{b n n}^{2} c_{n n n n} \\
(f): & \frac{T_{a b}^{2}}{\sigma(\sigma-\delta)^{2}} c_{a n n}^{2} c_{b n n}^{2} c_{n n(h-a-b)}^{2}
\end{aligned}
$$

Here $T_{a b}$ and $R_{a b}$ are the simple and double pole coefficients, and the coupling constants, in a lowest order computation, can be obtained from the lagrangian. For a complete comparison higher-order corrections should be included, as well as contributions from the subleading parts of the triangle and crossed box diagrams.

The exact S-matrix has a simple pole at $\sigma=\delta$. As we describe in Appendix A it is straightforward to check, using standard values of $T_{a b}$ and $R_{a b}$ and the above 
Figure 1: Contributions to the $S_{a b}$ amplitude of the $a_{2 n-1}^{(2)}$ theory which are responsible for the shift in the particle pole position. 
value for $\delta$ as well as the values of the coupling constants, that in the sum of the contributions (a), $2 \times(\mathrm{c}),(\mathrm{d})$ and (f) the pole at $\sigma=0$ cancels. Furthermore, to $O\left(\beta^{4}\right)$ the double pole cancels as well, leaving a simple pole at $\sigma=\delta$ with the correct $O\left(\beta^{2}\right)$ residue and indeed reproducing the result obtained from the exact S-matrix. The diagrams in Fig. 1.b,e contribute only at higher order. Thus, due to a subtle interplay between the location of the anomalous threshold poles and their residues, together with the value of the mass corrections, the simple particle pole in the S-matrix located at the (renormalized) particle mass has been cancelled, and replaced by simple pole at the location of the anomalous threshold singularity. Clearly, ordinary bootstrap ideas should not be applied in such a case and indeed, as we discussed in Refs. [8, 9], the charge conservation conditions for the corresponding vertex function $\left\langle\phi_{a} \phi_{b} \phi_{h-a-b}\right\rangle$ which would follow from the ordinary bootstrap do not hold.

It is interesting to contrast the above discussion of the anomalous poles with that in the $A^{(2)}(0,2 n-1)$ theory [5] which differs from our theory by the addition of one fermion: there, the bosonic field $\phi_{n}$ gives rise to the same anomalous threshold structure, but this is precisely cancelled by an identical structure from the fermion, so that the simple particle pole ends up in the position predicted by the mass formula (of the classical theory) and the usual bootstrap applies.

We turn now to the other anomalous threshold singularities and their identification with higher order poles in the S-matrix. As an aid to further discussion we note that many of the results and expressions derived in Ref. [5] for some of the amplitudes and the dual diagram constructions in the $A^{(2)}(0,2 n-1)$ theory can be taken over to our theory. Also, as mentioned above, the exact S-matrix elements $S_{a n}$ and $S_{a b}$ have the same form in the two theories, provided the blocks are reinterpreted according to our definitions above i.e. with $h \rightarrow H$.

We consider first the double poles in the amplitude $S_{a n}$ which occur at

$$
\theta=\frac{i \pi}{H}\left(\frac{H}{2}+2 p-a\right) \quad p=1,2, \ldots a-1
$$

In Ref. [5] we encountered similar double poles in the corresponding $S_{a n}$ amplitudes, and they were accounted for by both uncrossed and crossed box diagrams with dual diagrams such as those of Fig. 12 of that reference. Similar dual diagrams can be constructed in the present theory. Remarkably, although the construction is done with the renormalized masses, so that the lengths of the lines are different from what they were in the above reference, nonetheless the dual diagrams exist for both the crossed and the uncrossed boxes. The only change is in the location of the double poles, and this agrees with their location in the exact S-matrix. Indeed, let us consider the dual diagram in Fig. 2. We observe that in a triangle with sides $p, n, n$ (i.e. with lengths given by $\widetilde{m_{p}}, \widetilde{m_{n}}, \widetilde{m_{n}}$, ) the angle opposite the side $p$ is $\theta_{p}=\frac{2 \pi p}{H}$. This, and some elementary Euclidean geometry is sufficient to show that the dual diagram is planar, and that it predicts a double pole at the desired location. Additional contributions at the same location come from a crossed-box diagram. To one-loop order the coefficients of these double poles are the same as for the superalgebra case (where these contributions are also from bosons only), and 
Figure 2: Dual diagram for the double poles in $S_{a n}$ at $\theta=\frac{i \pi}{H}\left(\frac{H}{2}+2 p-a\right)$.

since our $S_{a n}$ has the same structure there is no need for any further checks at the one-loop level.

A new feature appears when we examine the double poles of the $S_{a b}$ amplitudes. For example, in the superalgebra case one obtained double poles from both an uncrossed and a crossed box, cf. Fig. 13 of reference [5] for the case of the $S_{n-1, n-1}$ amplitude. Now however, using the renormalized masses, the dual diagram for the uncrossed box can no longer be drawn in the plane and it would seem that although a double pole is still produced by the crossed box its coefficient would not match that of the exact S-matrix; the contribution from the uncrossed box is needed. The explanation is provided by realizing that in addition to the box diagram there are seemingly higher-order diagrams where one of the internal lines is replaced by the set of diagrams appearing in Fig. 1 which are responsible for the displacement of the single particle pole. In going through the Landau-Cutkoski analysis for the location and nature of singularities, one realizes then that a double pole is indeed produced, with the correct residue. Equivalently one can perform the dual diagram analysis using not the actual particle mass $\widetilde{m}_{h-a-b}^{2}$ but that corresponding to the pole of the S-matrix, namely $\widetilde{m}_{h-a-b}^{2}+\delta$. In this manner all the double poles of the exact S-matrix can be accounted for, and aside from verifying that the higher-order coefficients are correctly given we claim to have checked the self-consistency of the S-matrix we have constructed. 


\section{The $A^{(4)}(0,2 n)$ theory}

The classical lagrangian for the Toda theory based on the Lie superalgebra $A^{(4)}(0,2 n)$ is obtained from the lagrangian of the $a_{2 n-1}^{(2)}$ theory by dropping the field $\phi_{n}$ and adding a fermion:

$$
\begin{aligned}
\beta^{2} \mathcal{L} & =-\frac{1}{2} \sum_{1}^{n-1} \phi_{a} \square \phi_{a}+\frac{i}{2} \bar{\psi} \gamma \cdot \partial \psi \\
& -2 \sum_{k=2}^{n-1} \exp \left(-\sqrt{\frac{2}{h}} \sum_{1}^{n-1} m_{a} \cos \frac{(2 k-1) a \pi}{h} \phi_{a}\right)-\exp \left(-\sqrt{\frac{2}{h}} \sum_{1}^{n-1}(-1)^{a} m_{a} \phi_{a}\right) \\
& -2 \exp \left(-\sqrt{\frac{2}{h}} \sum_{1}^{n-1} m_{a} \cos \frac{a \pi}{h} \phi_{a}\right)-\frac{1}{\sqrt{2}} \bar{\psi} \psi \exp \left(-\frac{1}{\sqrt{2 h}} \sum_{1}^{n-1}(-1)^{a} m_{a} \phi_{a}\right)
\end{aligned}
$$

with bosonic masses and three-point couplings as in eq.(2.2,2.3), $m_{\psi}^{2}=2$ and $h=2 n-1$.

In the quantum theory normal-ordering is not sufficient to remove all infinities since, e.g., one-loop fermionic diagrams also lead to divergences. It appears that the correct prescription (which can be understood also by observing that such theories can be thought of as theories with explicitly broken supersymmetry [13]) is to normal-order the first and third exponentials, normal-order the exponential multiplying the fermions, and write the second exponential (which in the supersymmetric theory comes from eliminating an auxiliary field and is the partner of the last term) as the square of a normal-ordered exponential.

We recall that in the theory for the Lie superalgebra $A^{(2)}(0,2 n-1)$ which is obtained from the bosonic theory by adding the fermion but without dropping the $\phi_{n}$ boson, the one-loop mass corrections vanish. Here, having dropped the boson and introduced the fermion, the mass ratios $\left(m_{a} / m_{\psi}\right)^{2}$ receive one-loop corrections which have the same magnitude as those of the $a_{2 n-1}^{(2)}$ theory in eq.(2.4), but opposite sign.

One starts the S-matrix bootstrap by postulating an expression for the fermionfermion amplitude. Based on the previous case, and using the fact that the fermions couple to all the other particles in a manner similar to the couplings of $\phi_{n}$ we are led to

$$
S_{\psi \psi}=-\prod_{a=1}^{n-1} \frac{(2 a)_{H}(H-2 a)_{H}}{(2 a-B)_{H}(H-2 a+B)_{H}}
$$

with

$$
H=h-\frac{B}{2} \quad, \quad B=\frac{\beta^{2}}{2 \pi} \frac{1}{1+\frac{\beta^{2}}{4 \pi}}
$$

Some comments are in order. The above expression is very similar to the one for $S_{n n}$ in the $a_{2 n-1}^{(2)}$ theory and in fact one goes through exactly the same bootstrap procedure as before, with the following changes: we have put a minus sign in front of the product, to account for the fermionic nature of the particles, and we have 
dropped the block corresponding to $a=0$. In the bosonic theory that block was necessary essentially to account for a contribution to the S-matrix independent of $\theta$ and led us to choosing $\eta_{0}=-h \epsilon$ in eq.(2.24). Here we make instead the choice $\eta_{0}=0$ so that the S-matrix vanishes at $\theta=0$ (cf. the expression for the fermion amplitude $F(\theta)$ in eq.(2.5) of Ref. [5]). This choice leads to $H=h-\frac{B}{2}$ rather than $H=h+\frac{B}{2}$ which also agrees with the perturbation theory result that the mass corrections have an opposite sign. Beyond $O\left(\beta^{2}\right)$ the above expression for $B$ is conjectural.

The rest of the S-matrix elements are obtained again by standard bootstrap. We find that $S_{a \psi}$ is identical in form to the $S_{a n}$ of the $a_{2 n-1}^{(2)}$ theory and so is $S_{a b}$ (with the different value of $H$ however). As in the bosonic theory, for $a+b>n$, $S_{a b}$ has a simple pole shifted from the single-particle position to an anomalous threshold position. This time the relevant anomalous threshold singularity comes from a fermion loop (instead of the loop containing $\phi_{n}$ ) and the diagram in Fig. 1e is absent. The pole shift is somewhat different because of the change in sign implied by the new value of $H$. However, using the fact (which follows from cancellations between $\phi_{n}$ and $\psi$ contributions in the $A^{(2)}(0,2 n-1)$ theory) that now $\psi$ contributes $-T_{a b}$ and $-R_{a b}$ in eq.(2.40) while to lowest order the sign of $\delta$ changes, it is obvious that to $O\left(\beta^{4}\right)$ at least matters work in the same manner as for the bosonic theory. The various double poles can again be accounted for as before.

\section{The $b_{n}^{(1)}$ theory}

The Toda theory based on the nonsimply-laced affine Lie algebras $b_{n}^{(1)}$ is described by the lagrangian

$$
\begin{aligned}
\beta^{2} \mathcal{L} & =-\frac{1}{2} \sum_{1}^{n-1} \phi_{a} \square \phi_{a}-\frac{1}{2} \phi_{n} \square \phi_{n} \\
& -2 \sum_{k=2}^{n} \exp \left(-\sqrt{\frac{2}{h}} \sum_{1}^{n-1} m_{a} \cos \frac{(2 k-1) a \pi}{h} \phi_{a}\right) \\
& -\exp \left(-\sqrt{\frac{2}{h}} \sum_{1}^{n-1} m_{a} \cos \frac{a \pi}{h} \phi_{a}+\phi_{n}\right)-\exp \left(-\sqrt{\frac{2}{h}} \sum_{1}^{n-1} m_{a} \cos \frac{a \pi}{h} \phi_{a}-\phi_{n}\right)
\end{aligned}
$$

The Coxeter number is $h=2 n$. The masses and three-point couplings are given by the same formulas as in the $a_{2 n-1}^{(2)}$ theory, eqs.(2.2,2.3), but the fact that the Coxeter number is even leads to some significant differences. One-loop mass corrections turn out to have the same form as for the $a_{2 n-1}^{(2)}$ eq.(2.4), but opposite signs.

The S-matrix bootstrap starts as in the $a_{2 n-1}^{(2)}$ theory with the general Ansatz eq.(2.10) for $S_{n n}$. One goes through the bootstrap to obtain $S_{a n}$ and requires that it has a minimal number of poles. There are some changes in the procedure because the Coxeter number is even rather than odd. Again one is left with two possibilities. The one which leads to tree-level agreement with the amplitude obtained from the 
lagrangian gives

$$
S_{n n}=\prod_{a=1}^{n-1} \frac{(2 a)_{H}(H-2 a)_{H}}{(2 a-B)_{H}(H-2 a+B)_{H}} \frac{-1}{\left(\frac{B}{2}\right)_{H}\left(H-\frac{B}{2}\right)_{H}}
$$

with $H=h-\frac{B}{2}$. The minus sign in this relation between $H$ and $B$ is consistent with the sign of the one-loop mass shifts.

As compared with the previous situation, we observe that the product starts at $a=1$ and the two extra blocks have a slightly different form. An Ansatz which would start at $a=0$ leads to inconsistencies in the bootstrap, by introducing an additional pole. The other S-matrix elements $S_{a n}$ and $S_{a b}$ have the same form as before.

However there are important differences: as in the $a_{2 n-1}^{(2)}$ one must identify in $S_{a b}$ the poles corresponding to the exchanges of particles $\phi_{(a-b)}$ and $\phi_{(a+b)}$ or $\phi_{(h-a-b)}$. We are interested in particular in this last possibility, which occurs for $a+b>\frac{h}{2}$ and, for the $a_{2 n-1}^{(2)}$ theory, led to the displaced pole situation. The S-matrix element is

$$
\begin{aligned}
S_{a b}= & \prod_{p=1}^{b}\{2 p-b+a-1\}_{H}\{H-2 p+b-a+1\}_{H} \\
= & \frac{(a-b)_{H}(a-b+2)_{H}^{2} \cdots(a+b-2)_{H}^{2}(a+b)_{H}}{(a-b+B)_{H} \cdots \cdots \cdots \cdots(a+b-B)_{H}} \\
& \quad \times \frac{(H-a-b)_{H}(H-a-b+2)_{H}^{2} \cdots(H-a+b-2)_{H}^{2}(H-a+b)_{H}}{(H-a-b+B)_{H} \cdots \cdots \cdots \cdots \cdots \cdot(H-a+b-B)_{H}}
\end{aligned}
$$

It has a simple pole from a block $(a+b)$ which for $a+b>\frac{h}{2}$ is displaced with respect to the pole corresponding to the $s$-channel exchange of particle $\phi_{(h-a-b)}$. This is similar to the situation in the $a_{2 n-1}^{(2)}$ theory but here, since $h$ is even, there is a nearby double pole from the block $(H-2 p+b-a)_{H}^{2}$ for $p=\frac{h}{2}-a$. In Appendix A we describe how the nature and positions of these singularities can be explained. Other singularities can be accounted for in standard fashion. We note that were it not for the fact that $H \neq h$, one would encounter quadruple poles, but these are avoided because of the renormalization of the Coxeter number. 


\section{The $B^{(1)}(0, n)$ theory}

This Lie superalgebra Toda theory may be considered as a truncation of the two fermion $C^{(2)}(n+1)$ theory which was discussed in Ref. [5], obtained by dropping one of the fermions. The lagrangian, masses, and bosonic three-point couplings are given by

$$
\begin{aligned}
\beta^{2} \mathcal{L} & =-\frac{1}{2} \sum_{1}^{n} \phi_{a} \square \phi_{a}+\frac{i}{2} \bar{\psi} \gamma \cdot \partial \psi \\
& -2 \sum_{k=1}^{n-1} \exp \left(\sqrt{\frac{2}{h}} \sum_{1}^{n-1} m_{a} \cos \frac{a k \pi}{n} \phi_{a}-\sqrt{\frac{8}{h}}(-1)^{k} \phi_{n}\right) \\
& -\exp \left(\sqrt{\frac{2}{h}} \sum_{1}^{n-1} m_{a} \phi_{a}-\sqrt{\frac{8}{h}} \phi_{n}\right)-\exp \left(\sqrt{\frac{2}{h}} \sum_{1}^{n-1}(-1)^{a} m_{a} \phi_{a}-\sqrt{\frac{8}{h}}(-1)^{n} \phi_{n}\right) \\
& -\frac{1}{\sqrt{2}} \bar{\psi} \psi \exp \left(\frac{1}{\sqrt{2 h}} \sum_{1}^{n-1} m_{a} \phi_{a}-\sqrt{\frac{2}{h}} \phi_{n}\right)
\end{aligned}
$$

with

$$
\begin{array}{rlrl}
m_{\psi}^{2} & =2 \quad, \quad m_{a}^{2}=8 \sin ^{2}\left(\frac{a \pi}{h}\right) & a=1, \ldots, n \\
c_{a b c}=-\frac{\beta}{\sqrt{2 h}} m_{a} m_{b} m_{c}=-\frac{4 \beta}{\sqrt{h}} \Delta_{a b c} & \text { if } a \pm b \pm c=0 & \bmod h \\
c_{a b n}=\frac{\beta}{\sqrt{h}} m_{a} m_{b} m_{n}=\sqrt{2} \frac{4 \beta}{\sqrt{h}} \Delta_{a b n} & \text { if } a+b=n
\end{array}
$$

and all other bosonic three-point couplings are zero. The Coxeter number is $h=2 n$. The bosonic part of the lagrangian describes the nonsimply-laced $c_{n}^{(1)}$ affine Toda theory which we discuss below.

We begin the bootstrap with the Ansatz

$$
S_{\psi \psi}=\prod_{a=0}^{n-1} \frac{(2 a)_{H}(H-2 a)_{H}}{(2 a+B)_{H}(H-2 a-B)_{H}}\left(\frac{B}{2}\right)_{H}\left(H-\frac{B}{2}\right)_{H}
$$

This expression is the alternative choice one can make when constructing the Smatrix that led to $S_{n n}$ in the $b_{n}^{(1)}$ theory, with $H=h+\frac{B}{2}$ this time. It is not too difficult to check that it reproduces the correct tree-level result which can be extracted from that given in eq. (3.4) of Ref. [5] for the $C^{(2)}(n+1)$ theory,

The S-matrix element $S_{a \psi}$ has the same form as $S_{a n}$ in the previous cases and $S_{a b}$ also has the same form as before. Just as in the $b_{n}^{(1)}$ theory the analysis of pole and double pole singularities is affected by the fact that the Coxeter number is even.

As mentioned above, this theory is obtained from the $C^{(2)}(n+1)$ theory by dropping one of the fermions. In Ref. [7], we listed separate contributions to the mass corrections from the bosons and the fermions of that theory. Therefore, we can read off the one-loop mass corrections for the present theory:

$$
\delta \frac{m_{a}^{2}}{m_{n}^{2}}=-\frac{a \beta^{2}}{4 h^{2}} \sin \frac{2 a \pi}{h} \quad, \quad \delta \frac{m_{\psi}^{2}}{m_{n}^{2}}=0
$$


They are indeed in agreement with the corrections one can read from our S-matrix at $O\left(\beta^{2}\right)$. However, begining at $O\left(\beta^{4}\right)$ a new feature enters: the S-matrix predicts that the mass shifts of $\phi_{n}$ and $\psi$ are no longer equal, but in fact $\widetilde{m}_{n}<2 \widetilde{m}_{\psi}$. Therefore radiative corrections stabilize the particle $\phi_{n}$, the one-loop threshold effects discussed in Ref. [14 are absent at higher order, and the S-matrix bootstrap determines all the amplitudes, which was not the case in the $C^{(2)}(n+1)$ theory.

\section{The $c_{n}^{(1)}$ theory}

The lagrangian for this theory is obtained by dropping the fermion of the previous case. The Coxeter number is $h=2 n$ and the one loop mass corrections, from Ref. [7] are

$$
\delta \frac{m_{a}^{2}}{m_{n}^{2}}=-\frac{a \beta^{2}}{2 h^{2}} \sin \frac{2 a \pi}{h}
$$

Here one can start the bootstrap with the Ansatz

$$
S_{11}=\frac{(2)_{H}(H-2)_{H}}{(2-B)_{H}(H-2+B)_{H}} \cdot \frac{1}{(B)_{H}(H-B)_{H}}
$$

The first fraction accounts for the fact that $c_{11 a} \neq 0$ only if $a=2$ while the second fraction is required for the consistency of the bootstrap. Stepping up through the bootstrap one derives then

$$
S_{a b}=\prod_{p=1}^{b}\{2 p+a-b-1\}_{H}\{H-2 p-a+b+1\}_{H}
$$

This expression has too many higher order poles unless $H=h \pm B$. Comparison with the mass corrections gives $H=h+B$.

Our S-matrix is given by the same expression as the bosonic S-matrix for the $C^{(2)}(n+1)$ theory of Ref. [0], except for the replacement $h \rightarrow H$. This replacement has the effect of splitting the fourth-order poles in eq. (4.26) of that reference into pairs of double poles. These separated double poles can be accounted for as in Ref. [5], by the dual diagrams in Fig. 9a,b, and Fig. 9c,d respectively, of that reference. In addition, if $a+b>n$ one has again a displaced pole situation. We discuss this in Appendix A.

\section{The $d_{n}^{(2)}$ theory}

The lagrangian of this theory is obtained from the one for the $b_{n}^{(1)}$ theory by dropping particle $n$. The masses and 3-point couplings of the remaining particles are as in the $b_{n}^{(1)}$ theory. The Coxeter number is $h=2 n$. The S-matrix is constructed by the same bootstrap procedure as for the $c_{n}^{(1)}$ theory and has the form given in eq.(6.3) but now $H=h-B$. We note that compared to the previous case, the change in $H$ eliminates the pole which corresponded to the particle $\phi_{n}$ of that theory. 


\section{The $g_{2}^{(1)}$ theory}

Finally we give the S-matrix for the $g_{2}^{(1)}$ theory. Its lagrangian is

$$
\begin{aligned}
\beta^{2} \mathcal{L}= & -\frac{1}{2} \phi_{1} \square \phi_{1}-\frac{1}{2} \phi_{2} \square \phi_{2}-2 \exp \left(\sqrt{2} \phi_{2}\right) \\
& -3 \exp \left(\frac{1}{\sqrt{6}} \phi_{1}-\frac{1}{\sqrt{2}} \phi_{2}\right)-\exp \left(-\frac{\sqrt{3}}{\sqrt{2}} \phi_{1}-\frac{1}{\sqrt{2}} \phi_{2}\right)
\end{aligned}
$$

and the masses and three point couplings are

$$
\begin{gathered}
m_{1}^{2}=2 \quad, \quad m_{2}^{2}=6, \\
c_{111}=\sqrt{\frac{8}{3}} \beta \quad, \quad c_{112}=\sqrt{2} \beta \quad, \quad c_{222}=3 \sqrt{2} \beta .
\end{gathered}
$$

The Coxeter number is $h=6$.

In the $S_{11}$ amplitude both $\phi_{1}$ and $\phi_{2}$ appear as intermediate states. Because of the particular classical mass ratio, at the tree level the $s$-channel pole of one particle

coincides with the $u$-channel pole of the other particle. At the one-loop level the masses receive corrections given by

$$
\delta \frac{m_{2}^{2}}{m_{1}^{2}}=\beta^{2} \frac{1}{12 \sqrt{3}}
$$

which split the above poles. Therefore, starting the bootstrap with $S_{11}$ presents no problems if the physical masses are used in the S-matrix.

We begin with a general Ansatz for $S_{11}$ which implements this pole structure. Because of the $\phi_{1}$ self-coupling, $S_{11}$ has to satisfy the bootstrap consistency condition

$$
S_{11}(\theta)=S_{11}\left(\theta+\frac{1}{2} \theta_{11}^{1}\right) S_{11}\left(\theta-\frac{1}{2} \theta_{11}^{1}\right)
$$

with $\theta_{11}^{1}=\frac{2 \pi}{3}$. From $S_{11}$ we obtain $S_{12}$ by bootstrapping on the $\phi_{2}$ pole. The requirement that $S_{12}$ have the minimal number of blocks fixes the expression for $S_{11}$ uniquely and leads to

$$
\begin{aligned}
S_{11}(\theta)= & -\frac{(2)_{H}\left(4+\frac{2 B}{3}\right)_{H}(H-2)_{H}\left(H-4-\frac{2 B}{3}\right)_{H}}{\left(\frac{B}{3}\right)_{H}(2+B)_{H}\left(4+\frac{4 B}{3}\right)_{H}\left(H-\frac{B}{3}\right)_{H}(H-2-B)_{H}\left(H-4-\frac{4 B}{3}\right)_{H}} \\
S_{12}(\theta)= & \frac{(1)_{H}\left(3+\frac{2 B}{3}\right)_{H}(H-1)_{H}\left(H-3-\frac{2 B}{3}\right)_{H}}{(1+B)_{H}\left(3-\frac{B}{3}\right)_{H}(H-1-B)_{H}\left(H-3+\frac{B}{3}\right)_{H}} \\
S_{22}(\theta)= & -\frac{(2)_{H}\left(2+\frac{B}{3}\right)_{H}\left(2+\frac{2 B}{3}\right)_{H}}{(B)_{H}\left(2-\frac{B}{3}\right)_{H}(2+B)_{H}\left(2+\frac{4 B}{3}\right)_{H}} \\
& \times \frac{(H-2)_{H}\left(H-2-\frac{B}{3}\right)_{H}\left(H-2-\frac{2 B}{3}\right)_{H}}{(H-B)_{H}\left(H-2+\frac{B}{3}\right)_{H}(H-2-B)_{H}\left(H-2-\frac{4 B}{3}\right)_{H}}
\end{aligned}
$$


with $H=6+B$. We have checked tree-level agreement of these amplitudes. Again $B=\frac{\beta^{2}}{2 \pi}+O\left(\beta^{4}\right)$. The corrected masses are again given by the usual formula $m_{a}^{2}=$ $8 \sin ^{2} \frac{\pi}{H}$, in agreement with eq.(8.4).

In the absence of mass corrections $S_{12}$ would have an anomalous threshold double pole at $\theta=i \frac{\pi}{2}$ from a box diagram with internal $\phi_{1}$ lines. This double pole is split into the two simple poles from the blocks $\left(3+\frac{2 B}{3}\right)_{H}\left(H-3-\frac{2 B}{3}\right)_{H}$ of the exact Smatrix. We have checked consistency to $O\left(\beta^{4}\right)$. In the $S_{22}$ amplitude, there is again an interplay between the simple pole due to $\phi_{2}$ exchange and nearby anomalous threshold singularities.

\section{Discussion}

We have constructed in this paper elastic S-matrices for all the families of nonsimplylaced affine Toda theories. We summarize our results in Table 1. We have also constructed the S-matrix for $g_{2}^{(1)}$. We expect that the remaining three cases, namely $d_{4}^{(3)}, e_{6}^{(2)}$, and $f_{4}^{(1)}$ can be constructed in a similar manner.

The construction was carried out by paralleling that for the simply-laced theories, with one important difference: recognizing that radiative corrections shift the classical masses in a nontrivial manner, we allowed coupling-constant dependence in the numerator blocks $(x)$ of the usual construction. This led to the possibility of cancellations between numerator and denominator blocks and eliminated many unwanted poles that were produced in the course of the S-matrix bootstrap. In the end, by insisting on a minimal number of poles, we were left with very few possibilities, but enough to account for the S-matrices of all the families of nonsimply-laced Lie algebras $a_{2 n-1}^{(2)}, A^{(4)}(0,2 n), b_{n}^{(1)}, B^{(1)}(0, n), c_{n}^{(1)}$ and $d_{n}^{(2)}$. There are also a few isolated possibilities, which look slightly different, one of which we have shown to describe the Toda theory $g_{2}^{(1)}$.

These S-matrices do not satisfy all the usual bootstrap equations. In the simplylaced theories these equations are a simple consequence of the identification of all simple poles with elementary particle exchanges. In the nonsimply-laced theories however, the simple poles of $S_{a b}$ at $\theta=\frac{i \pi}{H}(a+b)$ for $a+b>\frac{h}{2}$ are not produced just by the single particle exchange of $\phi_{h-a-b}$ but involve additional multiparticle processes which give rise to anomalous threshold singularities. As a consequence, the usual bootstrap equations for these poles have to be replaced by modified ones which take into account these addtional singularities.

It is remarkable that our S-matrices have a fairly conventional form in terms of standard blocks, except for the replacement of the (integer) Coxeter number $h$ by a renormalized, coupling constant dependent $H$. In particular, this implies that quantum corrections lead to renormalized masses which are given by the usual classical formulas, but again with $h$ replaced by $H$.

The same feature persists for the higher spin conserved charges. We recall [3] 


\begin{tabular}{|c|c|c|c|c|c|c|}
\hline & $a_{2 n-1}^{(2)}$ & $\mathrm{A}^{(4)}(0,2 \mathrm{n})$ & $\mathbf{b}_{\mathbf{n}}^{(\mathbf{1})}$ & $\mathbf{B}^{(\mathbf{1})}(\mathbf{0}, \mathbf{n})$ & $\mathbf{c}_{\mathbf{n}}^{(\mathbf{1})}$ & $d_{n}^{(2)}$ \\
\hline$h$ & $2 n-1$ & $2 n-1$ & $2 n$ & $2 n$ & $2 n$ & $2 n$ \\
\hline$H$ & $h+\frac{B}{2}$ & $h-\frac{B}{2}$ & $h-\frac{B}{2}$ & $h+\frac{B}{2}$ & $h+B$ & $h-B$ \\
\hline$m_{a}^{2}$ & $8 \sin ^{2}\left(\frac{a \pi}{h}\right)$ & $8 \sin ^{2}\left(\frac{a \pi}{h}\right)$ & $8 \sin ^{2}\left(\frac{a \pi}{h}\right)$ & $8 \sin ^{2}\left(\frac{a \pi}{h}\right)$ & $8 \sin ^{2}\left(\frac{a \pi}{h}\right)$ & $8 \sin ^{2}\left(\frac{a \pi}{h}\right)$ \\
\hline$m_{n}^{2}$ & 2 & - & 2 & $8 \sin ^{2}\left(\frac{n \pi}{h}\right)$ & $8 \sin ^{2}\left(\frac{n \pi}{h}\right)$ & - \\
\hline$m_{\psi}^{2}$ & - & 2 & - & 2 & - & - \\
\hline$\epsilon_{a b(a+b)}$ & 1 & 1 & 1 & -1 & -1 & 1 \\
\hline$\epsilon_{a b(h-a-b)}$ & -1 & -1 & -1 & -1 & -1 & -1 \\
\hline$\epsilon_{n a(n-a)}$ & 0 & 0 & 0 & $\sqrt{2}$ & $\sqrt{2}$ & 0 \\
\hline$\epsilon_{n n a}$ & 1 & 0 & 1 & 0 & 0 & 0 \\
\hline$S_{a b}$ & $P_{a b}$ & $P_{a b}$ & $P_{a b}$ & $P_{a b}$ & $P_{a b}$ & $P_{a b}$ \\
\hline$S_{a n}$ & $Q_{a}$ & - & $Q_{a}$ & $P_{a n}$ & $P_{a n}$ & - \\
\hline$S_{n n}$ & $\{B\}_{H}\{H-B\}$ & - & $\frac{B}{\left.\frac{B}{2}\right\}_{H}\left\{H-\frac{B}{2}\right\}}$ & $P_{n n}$ & $P_{n n}$ & - \\
\hline$S_{a \psi}$ & - & $Q_{a}$ & - & $Q_{a}$ & - & - \\
\hline$S_{n \psi}$ & - & - & - & $Q_{n}$ & - & - \\
\hline$S_{\psi \psi}$ & - & $R_{-}$ & - & $\frac{\left(\frac{B}{2}\right)_{H}\left(H-\frac{B}{2}\right)_{H}}{(B)_{H}(H-B)_{H}}$ & - & - \\
\hline
\end{tabular}

$$
\begin{aligned}
c_{i j k} & =\epsilon_{i j k} \frac{4 \beta}{\sqrt{h}} \Delta_{i j k} \\
P_{i j} & =\prod_{p=1}^{j}\{i-j-1+2 p\}_{H}\{H-i+j+1-2 p\}_{H} \\
Q_{i} & =\prod_{p=1}^{i}\left\{\frac{H}{2}-i-1+2 p\right\}_{H} \quad R_{ \pm}=-\prod_{p=1}^{n-1} \frac{(2 p)_{H}(H-2 p)_{H}}{(2 p \pm B)_{H}(H-2 p \mp B)_{H}}
\end{aligned}
$$

Table 1: The classical masses, 3-point couplings, and S-matrices of the nonsimplylaced affine Toda theories. 
that they are obtained in terms of the Fourier coefficients of the matrix

$$
\begin{aligned}
\varphi_{a b}(\theta) & \equiv i \frac{d}{d \theta} \ln S_{a b}(\theta) \\
& =\sum_{s=0}^{\infty} \varphi_{a b}^{(s)} e^{-s|\theta|}
\end{aligned}
$$

Each row and column of this matrix satisfies the charge conservation equation, as can be seen by inserting the Fourier expansion into the logarithmic derivative of the S-matrix bootstrap equation:

$$
\varphi_{c d}^{(s)}=\varphi_{a d}^{(s)} e^{-s \bar{\theta}_{a c}^{b}}+\varphi_{b d}^{(s)} e^{s \bar{\theta}_{b c}^{a}}
$$

(Note however that this relation will be modified in those situations where the usual S-matrix bootstrap equation is not valid due to anomalous threshold effects, as discussed in [8, 9].)

For the $a_{2 n-1}^{(2)}$ and $b_{n}^{(1)}$ theories we find that the even $s$ charges vanish, while for odd $s$ we obtain for the charges $\gamma^{(s)}$

$$
\frac{\gamma_{a}^{(s)}}{\gamma_{n}^{(s)}}=\frac{\varphi_{a b}^{(s)}}{\varphi_{n b}^{(s)}}=(-1)^{\frac{s-1}{2}} 2 \sin \frac{\pi s a}{H}
$$

At the one-loop level this agrees with the explicit values calculated for the spin 3 charges of the $a_{3}^{(2)}$ theory in Refs. 8, 9]. For the $c_{n}^{(1)}$ and $d_{n}^{(2)}$ theories we obtain

$$
\frac{\gamma_{a}^{(s)}}{\gamma_{n}^{(s)}}=\frac{\sin \frac{\pi s a}{H}}{\sin \frac{\pi s n}{H}}
$$

and for the $A^{(4)}(0,2 n)$ and $B^{(1)}(0, n)$ theories

$$
\frac{\gamma_{a}^{(s)}}{\gamma_{\psi}^{(s)}}=\frac{\varphi_{a b}^{(s)}}{\varphi_{\psi b}^{(s)}}=(-1)^{\frac{s-1}{2}} 2 \sin \frac{\pi s a}{H}
$$

For $s=1$ these formulas do of course reproduce the formulas for the renormalized masses. Because of the replacement of $h$ by $H$, the usual fact that the charges are eigenvalues of the incidence matrix [15] no longer holds.

We have checked by comparing the exact S-matrices to one-loop perturbative calculations that to $O\left(\beta^{4}\right) B=\frac{\beta^{2}}{2 \pi}\left(1+\frac{\beta^{2}}{4 \pi}\right)^{-1}$. For the simply-laced theories, it is well known that this particular coupling-constant dependence implies invariance under the weak-coupling strong-coupling exchange $\beta \rightarrow \frac{4 \pi}{\beta}$. This exchange implies $B \rightarrow 2-B$ and leaves invariant the product $(x-1+B)(x+1-B)$ which appears in $\{x\}$. Here the situation is somewhat different because of the additional couplingconstant dependence in $H$.

Let us consider the $a_{2 n-1}^{(2)}$ theory, with the renormalized Coxeter number $H_{(a)}=$ $2 n-1+\frac{B}{2}$. Under the above substitution

$$
H_{(a)} \rightarrow 2 n-\frac{B}{2}=H_{(b)}
$$


where $H_{(b)}$ is the renormalized Coxeter number for the $b_{n}^{(1)}$ theory. Furthermore, under the above substitution we have, starting from the $a_{2 n-1}^{(2)}$ theory

$$
\begin{aligned}
S_{n n}^{(a)}= & \prod_{a=0}^{n-1} \frac{(2 a)_{H}(H-2 a)_{H}}{(2 a+B)_{H}(H-2 a-B)_{H}} \\
& \rightarrow \prod_{a=0}^{n-1} \frac{(2 a)_{H}(H-2 a)_{H}}{(2(a+1)-B)_{H}(H-2(a+1)+B)_{H}} \\
= & -\prod_{a=1}^{n-1} \frac{(2 a)_{H}(H-2 a)_{H}}{(2 a-B)_{H}(H-2 a+B)_{H}} \cdot \frac{1}{\left(\frac{B}{2}\right)_{H}\left(H-\frac{B}{2}\right)_{H}}=S_{n n}^{(b)}
\end{aligned}
$$

where for notational simplicity we have omitted the subscripts on the $H$. Since all other S-matrix elements are determined by the bootstrap, this shows that under the substitution $\beta \rightarrow \frac{4 \pi}{\beta}$ the S-matrices for the $a_{2 n-1}^{(2)}$ and $b_{n}^{(1)}$ theories go into each other. In the same manner one can check that the S-matrices for $c_{n}^{(1)}$ and $d_{n+1}^{(2)}$ go into each other, as do the S-matrices for the $A^{(4)}(0,2 n)$ and $B^{(1)}(0, n-1)$ theories. In particular, at the symmetric point, $\beta^{2}=4 \pi$ these theories are pairwise equivalent.

We comment on two other features: first, application of the thermodynamic Bethe Ansatz [15] leads to the prediction of the central charge in the conformal limit, $c=c_{\text {free }}$, as one might expect. Second, it does not appear that a "minimal" S-matrix exists for these theories. One cannot drop the blocks involving the dependence on the coupling constant through the parameter $B$ since these are required for the consistency of the bootstrap.

\section{A The displaced poles}

In the main text we have drawn the reader's attention to the presence of simple poles in the exact S-matrix which are not located precisely at the expected position corresponding to certain particle masses. As outlined for the case of the $a_{2 n-1}^{(2)}$ theory, the shift in pole positions is due to the presence of nearby anomalous threshold singularities. Because these shifted poles are a novel feature and because we have claimed that they lead to breakdown of the usual bootstrap procedure, it is important to verify that all such poles can be explained by a diagram analysis. In this Appendix we present details of this perturbative verification.

In all the theories that we have considered, the amplitude $S_{a b}$ has the universal form (choosing $a>b$ )

$$
\begin{aligned}
S_{a b}= & \prod_{p}\{2 p-b+a-1\}_{H}\{H-2 p+b-a+1\}_{H} \\
= & \{a-b+1\}_{H}\{a-b+3\}_{H} \cdots\{a+b-1\}_{H} \\
& \times\{H-a-b+1\}_{H} \cdots\{H-a+b-1\}_{H}
\end{aligned}
$$

It has simple poles from blocks $(a-b)_{H}$ and $(H-a-b)_{H}$ corresponding to the exchange of particle $\phi_{a-b}$ and from blocks $(a+b)_{H}$ and $(H-a-b)_{H}$ which in the case 
$a+b \leq \frac{h}{2}$ correspond to the exchange of particle $\phi_{a+b}$. In the case $a+b>\frac{h}{2}$ however, where the couplings of the Toda theories show that particle $\phi_{h-a-b}$ is exchanged, these poles are not at the expected position.

We study the poles of $S_{a b}$ in the neighborhood of the point where the $s$-channel pole of particle $\phi_{(h-a-b)}$ should appear. Such poles come from the block $\{a+b-1\}_{H}$. If $h$ is even, the above product also contains the blocks $\{H-(h-a-b)-1\}_{H}\{H-$ $(h-a-b)+1\}_{H}$ and these too contribute nearby poles. We will write formulas for this case, with the understanding that corresponding contributions should be omitted for those theories where $h$ is odd. Thus we will consider singularities arising from

$$
S_{a b} \sim \frac{(a+b)_{H}}{(a+b-B)_{H}} \cdot \frac{\left[(a+b+H-h)_{H}\right]^{2}}{(a+b+H-h-B)_{H}(a+b+H-h+B)_{H}}
$$

To the order of our calculation the remaining blocks in $S_{a b}$ reduce to unity at the poles.

The pole from the block $(a+b)_{H}$ corresponds to a position in the $s$-plane

$$
\begin{aligned}
\widetilde{m}_{a}^{2}+\widetilde{m}_{b}^{2}+2 \widetilde{m}_{a} \widetilde{m}_{b} \cos \frac{\pi}{H}(a+b) & =8 \sin ^{2} \frac{\pi}{H}(a+b) \\
& =\widetilde{m}_{(h-a-b)}^{2}+\delta
\end{aligned}
$$

where, to lowest order in $\beta^{2}$ (cf. eq.(2.40))

$$
\delta=8 \frac{\pi}{H}(H-h) \Delta_{c} \quad, \quad \Delta_{c}=-\sin \frac{2 \pi}{H}(a+b)
$$

The pole from $(a+b+H-h)_{H}$ corresponds to a position

$$
\widetilde{m}_{a}^{2}+\widetilde{m}_{b}^{2}+2 \widetilde{m}_{a} \widetilde{m}_{b} \cos \frac{\pi}{H}(a+b+H-h)=\widetilde{m}_{(h-a-b)}^{2}+\hat{\delta}
$$

where

$$
\hat{\delta}=-4 \frac{\pi}{H}(H-h) \hat{\Delta}_{c} \quad, \quad \hat{\Delta}_{c}=4 \sin \frac{\pi}{H}(a+b) \cos \frac{\pi a}{H} \cos \frac{\pi b}{H}
$$

Defining also

$$
\sigma=s-\widetilde{m}_{(h-a-b)}^{2}
$$

so that the actual particle pole is at $\sigma=0$, the poles are located at $\sigma=\delta$ and $\sigma=\hat{\delta}$ respectively.

The coefficients of the poles are obtained from the general expansions

$$
\begin{aligned}
\frac{(x)}{(x+\gamma)}= & \frac{1}{\operatorname{sh}\left(\frac{\theta}{2}-\frac{\theta_{0}}{2}\right)}\left(-\frac{i \pi}{2 h} \gamma+O\left(\gamma^{2}\right)\right)+\text { regular terms } \\
\frac{(x)^{2}}{\left(x+\gamma_{1}\right)\left(x+\gamma_{2}\right)}= & \frac{1}{\operatorname{sh}\left(\frac{\theta}{2}-\frac{\theta_{0}}{2}\right)}\left(-\frac{i \pi}{2 h}\left(\gamma_{1}+\gamma_{2}\right)+O\left(\gamma^{2}\right)\right) \\
& +\frac{1}{\operatorname{sh}^{2}\left(\frac{\theta}{2}-\frac{\theta_{0}}{2}\right)}\left(\left(\frac{i \pi}{2 h}\right)^{2} \gamma_{1} \gamma_{2}+O\left(\gamma^{3}\right)\right)+\text { regular terms }
\end{aligned}
$$


We will also use $\operatorname{sh}\left(\frac{\theta}{2}-\frac{\theta_{0}}{2}\right) \sim \frac{1}{2}\left(\theta-\theta_{0}\right) \sim-\frac{i}{8 \Delta}\left(s-s_{0}\right)$ where

$$
\Delta=4 \sin \frac{\pi a}{H} \sin \frac{\pi b}{H} \sin \frac{\pi(a+b)}{H}
$$

For comparison with perturbation theory it is also convenient to remove the Jacobian and external particle normalization by multiplying with the factor $4 i m_{a} m_{b} \operatorname{sh} \theta \sim$ $8 i \Delta$. Finally, to the order that we are working with, $B \sim \frac{\beta^{2}}{2 \pi}$.

We display next the S-matrix poles theory by theory:

$\mathbf{a}_{\mathbf{2 n}-\mathbf{1}}^{(\mathbf{2})}$ : here $H-h=\frac{B}{2}$ and $h=2 n-1$ is odd so that the S-matrix has only a simple pole

$$
S_{a b} \sim \frac{F}{\sigma-\delta}
$$

with

$$
F=-16 i \frac{\beta^{2}}{h} \Delta^{2} \quad, \quad \delta=2 \frac{\beta^{2}}{h} \Delta_{c}
$$

$\mathbf{A}^{(4)}(\mathbf{0}, \mathbf{2 n})$ : here $H-h=-\frac{B}{2}$ and $\delta=-2 \frac{\beta^{2}}{h} \Delta_{c}$. Otherwise the situation is the same as in the previous case.

$\mathbf{b}_{\mathbf{n}}^{(\mathbf{1})}$ : here $H-h=-\frac{B}{2}$ and $h=2 n$ is even. We obtain

$$
S_{a b} \sim \frac{F}{\sigma-\delta}+\frac{G}{(\sigma-\hat{\delta})^{2}}+\frac{K}{(\sigma-\delta)(\sigma-\hat{\delta})^{2}}
$$

where

$$
\begin{aligned}
F & =-16 i \frac{\beta^{2}}{h} \Delta^{2}, \quad G=-32 i \frac{\beta^{4}}{h^{2}} \Delta^{3}, \quad K=64 i \frac{\beta^{6}}{h^{3}} \Delta^{4} \\
\delta & =-2 \frac{\beta^{2}}{h} \Delta_{c}, \quad \hat{\delta}=\frac{\beta^{2}}{h} \hat{\Delta}_{c} .
\end{aligned}
$$

$\mathbf{B}^{(\mathbf{1})}(\mathbf{0}, \mathbf{n})$ : this is similar to the $b_{n}^{(1)}$ case, but with $H-h=\frac{B}{2}, \delta=2 \frac{\beta^{2}}{h} \Delta_{c}$ and $\hat{\delta}=-\frac{\beta^{2}}{h} \hat{\Delta}_{c}$.

$\mathbf{c}_{\mathbf{n}}^{(\mathbf{1})}$ : here $H-h=B$ and $h=2 n$ is even. We observe that in eq. (A.2) the pole from the block $(a+b)_{H}$ cancels against a zero from the denominator block $(a+b+H-h-B)_{H}$ so that we have now

$$
S_{a b} \sim \frac{F}{\sigma-\hat{\delta}}+\frac{G}{(\sigma-\hat{\delta})^{2}}
$$

with

$$
F=-16 i \frac{\beta^{2}}{h} \Delta^{2}, \quad G=-64 i \frac{\beta^{4}}{h^{2}} \Delta^{3}, \quad \hat{\delta}=-2 \frac{\beta^{2}}{h} \hat{\Delta}_{c}
$$

$\mathbf{d}_{\mathbf{n}}^{(2)}$ : here $H-h=-B$ so that again the simple pole from $(a+b)_{H}$ gets canceled, but also the double pole from $\left[(a+b+H-h)_{H}\right]^{2}$ gets reduced to a simple pole. We have

$$
S_{a b} \sim \frac{F}{\sigma-\hat{\delta}}
$$


Figure 3: Dual diagrams for anomalous threshold poles in $S_{a b}$.

with

$$
F=-16 i \frac{\beta^{2}}{h} \Delta^{2}, \quad \hat{\delta}=2 \frac{\beta^{2}}{h} \hat{\Delta}_{c}
$$

The pole structure for each theory will be accounted for by an interplay between single particle poles and anomalous threshold poles, as we verify case by case. For the analysis the two dual diagrams in Fig. 3 associated with anomalous threshold singularities of triangle and box diagrams that we encounter below are relevant. The interested reader should consult Ref. [5] for an explanation of the notation and procedure. We list first the geometrical quantities of interest:

for Fig. 3.a

$$
\begin{aligned}
\Delta_{a} & =\sin \frac{2 \pi}{H} a \quad, \quad \Delta_{b}=\sin \frac{2 \pi}{H} b \\
\Delta & =4 \sin \frac{\pi}{H} a \sin \frac{\pi}{H} b \sin \frac{\pi}{H}(a+b) \\
\Delta_{c} & =\Delta-\Delta_{a}-\Delta_{b}=-\sin \frac{2 \pi}{H}(a+b)
\end{aligned}
$$

for Fig. 3.b

$$
\begin{aligned}
\hat{\Delta}_{a} & =-4 \sin \frac{\pi}{H} a \cos \frac{\pi}{H} b \cos \frac{\pi}{H}(a+b) \\
\hat{\Delta}_{b} & =-4 \sin \frac{\pi}{H} b \cos \frac{\pi}{H} a \cos \frac{\pi}{H}(a+b) \\
\Delta & =4 \sin \frac{\pi}{H} a \sin \frac{\pi}{H} b \sin \frac{\pi}{H}(a+b) \\
\hat{\Delta}_{c} & =\Delta-\hat{\Delta}_{a}-\hat{\Delta}_{b}=4 \sin \frac{2 \pi}{H}(a+b) \cos \frac{2 \pi}{H} a \cos \frac{2 \pi}{H} b
\end{aligned}
$$

All the couplings, pole residues and pole shifts are expressible in terms of the areas of the various triangles. 
Figure 4: Feynman diagrams leading to the shift of the $\phi_{h-a-b}$-pole. Diagrams b),c) and d) contribute only in $a_{2 n-1}^{(2)}$ and $b_{n}^{(1)}$.

We consider now the Feynman diagrams which lead to poles at the relevant locations.

$$
\text { Fig. 4.a: } \quad \frac{T_{1}}{\sigma} \quad, \quad T_{1}=-i c_{a b(h-a-b)}^{2}=-16 i \frac{\beta^{2}}{h} \Delta^{2}
$$

This diagram, which corresponds to the actual s-channel exchange of particle $\phi_{h-a-b}$, gives the same contribution in all the theories.

The diagrams in Fig. 田b,c,d exist only in those theories with $c_{a n n}$ couplings, i.e. in $a_{2 n-1}^{(2)}$ and $b_{n}^{(1)}$ and give the following contributions:

$$
\text { Fig. 4.b: } \frac{T_{2}}{\sigma(\sigma-\delta)} \quad, \quad T_{2}=-64 i \frac{\beta^{4}}{h^{2}} \Delta^{2} \Delta_{c}
$$

Here $T_{2}$ has been computed by including coupling constant factors and the residue of the anomalous threshold pole of the triangle diagram derived in eq.(4.23) of Ref. [5]. We have also included a factor of two for the interchange of the two vertices.

$$
\text { Fig. 4.c: } \quad \frac{T_{3}}{(\sigma-\delta)^{2}} \quad, \quad T_{3}=32 i \frac{\beta^{4}}{h^{2}} \Delta^{2} \Delta_{c}
$$

Here $T_{3}$ includes coupling constant factors and the coefficient $R_{2}$ of the crossed box diagram anomalous threshold double pole, as given in eq.(4.18) of Ref. [5].

$$
\text { Fig. 4.d: } \quad \frac{T_{4}}{\sigma(\sigma-\delta)^{2}} \quad, \quad T_{4}=-64 i \frac{\beta^{6}}{h^{3}} \Delta^{2} \Delta_{c}^{2}
$$

with $T_{4}$ including coupling constant factors and the square of the residue of the triangle anomalous threshold pole.

The diagrams in Fig. 5 exist only if $h=2 n$, i.e. for the $b, B, c$ and $d$ theories. We have

$$
\text { Fig. 5.a: } \frac{\hat{T}_{2}}{\sigma(\sigma-\hat{\delta})} \quad, \quad \hat{T}_{2}=\mp 64 i \frac{\beta^{4}}{h^{2}} \Delta^{2} \Delta_{c}
$$


Figure 5: Feynman diagrams leading to the shift of the $\phi_{h-a-b}$-pole in theories where $h=2 n$.

Figure 6: Feynman diagrams leading to the shift of the $\phi_{h-a-b}$-pole. Diagrams a) exists in $c_{n}^{(1)}$ and diagram b) in $b_{n}^{(1)}$.

with the plus sign for the $c_{n}^{(1)}$ theory only;

$$
\begin{array}{ccc}
\text { Fig. 5.b: } & \frac{\hat{T}_{3}}{(\sigma-\hat{\delta})^{2}} \quad, \quad \hat{T}_{3}=32 i \frac{\beta^{4}}{h^{2}} \Delta^{2} \hat{\Delta}_{c} \\
\text { Fig. 5.c: } & \frac{\hat{T}_{4}}{\sigma(\sigma-\hat{\delta})^{2}} \quad, \quad \hat{T}_{4}=-64 i \frac{\beta^{6}}{h^{3}} \Delta^{2} \hat{\Delta}_{c}^{2}
\end{array}
$$

The diagrams in Fig. 6.a exists only if $h=2 n$ and if in addition the coupling $c_{a n(n-a)}$ exists, i.e. only for the $c_{n}^{(1)}$ theories. They give

$$
\text { Fig.6a: } \quad \frac{\tilde{T}_{3}}{(\sigma-\hat{\delta})^{2}} \quad, \quad \tilde{T}_{3}=-64 i\left(\Delta^{3}+\Delta^{2} \hat{\Delta}_{c}\right)
$$

The diagram in Fig. Gb exists only if $h=2 n$ and $c_{a n n}$ exists, i.e. only for $b_{n}^{(1)}$. 
Including a factor of two for the interchange of the two triangles, we have

$$
\text { Fig.6b: } \quad \frac{\tilde{T}_{4}}{\sigma(\sigma-\delta)(\sigma-\hat{\delta})} \quad, \quad \tilde{T}_{4}=-128 i \frac{\beta^{6}}{h^{3}} \Delta^{2} \Delta_{c} \hat{\Delta}_{c}
$$

We consider now each theory, and compare the pole contributions from the Smatrix with the corresponding poles of Feynman diagrams.

$\mathbf{a}_{\mathbf{2 n - 1}}^{(\mathbf{2})}$ : we want to show that

$$
\frac{F}{\sigma-\delta}=\frac{T_{1}}{\sigma}+\frac{T_{2}}{\sigma(\sigma-\delta)}+\frac{T_{3}}{(\sigma-\delta)^{2}}+\frac{T_{4}}{\sigma(\sigma-\delta)^{2}}
$$

This requires

$$
\begin{aligned}
& T_{1}=F \\
& 2 \delta T_{1}-T_{2}-T_{3}=\delta F \\
& \delta^{2} T_{1}-\delta T_{2}+T_{4}=0
\end{aligned}
$$

Using the expressions in eqs. A.11, A.20, A.21, A.22 and A.23) one can check that these conditions are indeed satisfied.

$\mathbf{b}_{\mathbf{n}}^{(\mathbf{1})}$ : we have to show that

$$
\begin{gathered}
\frac{F}{\sigma-\delta}+\frac{G}{(\sigma-\hat{\delta})^{2}}+\frac{K}{(\sigma-\delta)(\sigma-\hat{\delta})^{2}}=\frac{T_{1}}{\sigma}+\frac{T_{2}}{\sigma(\sigma-\delta)}+\frac{T_{3}}{(\sigma-\delta)^{2}} \\
+\frac{T_{4}}{\sigma(\sigma-\delta)^{2}}+\frac{\hat{T}_{2}}{\sigma(\sigma-\hat{\delta})}+\frac{\hat{T}_{3}}{(\sigma-\hat{\delta})^{2}}+\frac{\hat{T}_{4}}{\sigma(\sigma-\hat{\delta})^{2}}+\frac{\tilde{T}_{4}}{\sigma(\sigma-\delta)(\sigma-\hat{\delta})}
\end{gathered}
$$

from which we derive the following conditions:

$$
\begin{array}{ll}
\text { a) } & T_{1}=F \\
\text { b) } \quad & \delta T_{1}-T_{2}-\hat{T}_{2}-T_{3}-\hat{T}_{3}=-G \\
\text { c) } \quad & \left(-\delta^{2}+2 \delta \hat{\delta}\right) T_{1}+(\delta-2 \hat{\delta}) T_{2}-\hat{\delta} \hat{T}_{2}+(-2 \hat{\delta}+2 \delta) T_{3} \\
& \quad+T_{4}+\hat{T}_{4}+\tilde{T}_{4}=K \\
\text { d) } \quad\left(-\delta \hat{\delta}^{2}-2 \delta^{2} \hat{\delta}+\delta^{3}\right) T_{1}+\left(\hat{\delta}^{2}+2 \delta \hat{\delta}-\delta^{2}\right) T_{2}+2 \delta \hat{\delta} \hat{T}_{2} \\
\quad \\
\quad+\left(\hat{\delta}^{2}-\delta^{2}\right) T_{3}-2 \hat{\delta} T_{4}-2 \delta \hat{T}_{4}-(\delta+\hat{\delta}) \tilde{T}_{4}=-\delta K \\
\quad \text { e) } \quad \delta^{2} \tilde{\delta}^{2} T_{1}-\delta \hat{\delta}^{2} T_{2}-\delta^{2} \hat{\delta} \hat{T}_{2}+\hat{\delta}^{2} T_{4}+\delta^{2} \hat{T}_{4}+\delta \hat{\delta} \tilde{T}_{4}=0
\end{array}
$$

With the expressions given earlier, it is not too difficult to check that the conditions a), b) and e) are indeed satisfied, as a result of the interplay between "hatted" and "unhatted" diagrams using also the identity $2 \Delta_{c}+\hat{\Delta}_{c}=\Delta$, but that the conditions c) and d) are not. However, there are additional two-loop diagrams that must be considered in this case: the one in Fig. 7.a and the one obtained by interchanging top and bottom rungs. The corresponding dual diagram in Fig. 77.b would be planar and therefore lead to a triple pole if the classical masses were used. If one uses the actual masses the diagram is not quite planar, but those dual diagrams associated with reduced diagrams are, leading to a splitting of the triple pole into a simple and 
Figure 7: An additional diagram contributing in the $b_{n}^{(1)}$ theory. a) Feynman diagram, b) Dual diagram.

a double pole. Although we have not checked the details, it is plausible that one obtains a contribution

$$
\frac{T_{5}}{(\sigma-\delta)^{2}(\sigma-\hat{\delta})}+\frac{\hat{T}_{5}}{(\sigma-\delta)(\sigma-\hat{\delta})^{2}}
$$

with

$$
T_{5}=64 i \frac{\beta^{6}}{h^{3}} \Delta^{3} \Delta_{c} \quad, \quad \hat{T}_{5}=64 i \frac{\beta^{6}}{h^{3}} \Delta^{3}\left(\Delta_{c}+\hat{\Delta}_{c}\right) .
$$

Including this contribution does not affect the three conditions that were satisfied previously, but leads now to satisfaction of the other two, and therefore an explanation of the pole structure for the $b_{n}^{(1)}$ theory.

$\mathbf{c}_{\mathbf{n}}^{(\mathbf{1})}$ : we must show that

$$
\frac{F}{(\sigma-\hat{\delta})}+\frac{G}{(\sigma-\hat{\delta})^{2}}=\frac{T_{1}}{\sigma}+\frac{\hat{T}_{2}}{\sigma(\sigma-\hat{\delta})}+\frac{\hat{T}_{3}}{(\sigma-\hat{\delta})^{2}}+\frac{\hat{T}_{4}}{\sigma(\sigma-\hat{\delta})^{2}}+\frac{\tilde{T}_{3}}{(\sigma-\hat{\delta})^{2}}
$$

leading to the conditions

$$
\begin{aligned}
& T_{1}=F \\
& 2 \hat{\delta} T_{1}-\hat{T}_{2}-\hat{T}_{3}-\tilde{T}_{3}=\hat{\delta} F-G \\
& \hat{\delta}^{2} T_{1}-\hat{\delta} \hat{T}_{2}+\hat{T}_{4}=0
\end{aligned}
$$

which are indeed satisfied.

$\mathbf{d}_{\mathbf{n}}^{(2)}$ : we must show

$$
\frac{F}{\sigma-\hat{\delta}}=\frac{T_{1}}{\sigma}+\frac{\hat{T}_{2}}{\sigma(\sigma-\hat{\delta})}+\frac{\hat{T}_{3}}{(\sigma-\hat{\delta})^{2}}+\frac{\hat{T}_{4}}{\sigma(\sigma-\hat{\delta})^{2}}
$$


leading to

$$
\begin{aligned}
& T_{1}=F \\
& \hat{\delta} T_{1}-\hat{T}_{2}-\hat{T}_{3}=0 \\
& \hat{\delta}^{2} T_{1}-\hat{\delta} \hat{T}_{2}+\hat{T}_{4}=0
\end{aligned}
$$

which are again satisfied.

This completes the perturbative verification of the position and residues of the displaced poles in these bosonic exact S-matrices. We have not examined in detail the corresponding situation for the theories with fermions or for $g_{2}^{(1)}$.

\section{B The coupling constant dependence}

For the simply-laced theories it has been checked in perturbation theory [12] that the coupling constant dependence of the S-matrix arises through the combination $B=\frac{1}{2 \pi} \frac{\beta^{2}}{1+\frac{\beta^{2}}{4 \pi}}$. In this appendix we carry out a similar perturbative verification, at the one-loop level, for two nonsimply-laced cases. We compute from the lagrangian, to $O\left(\beta^{4}\right)$, the residue of a suitable S-matrix element at a single-particle pole, and compare to the same expression obtained from the exact S-matrix. To make the calculations manageable, we restrict ourselves to cases with only two fields.

We consider first the $a_{3}^{(2)}$ theory, with fields $\phi_{1}$ and $\phi_{2}$, classical masses $m_{1}^{2}=6$, $m_{2}^{2}=2$, and relevant couplings

$$
\mathcal{L}_{\text {int }}=-\phi_{1}^{3}+\phi_{1} \phi_{2}^{2}-\frac{3}{4} \phi_{1}^{4}-\frac{1}{2} \phi_{1}^{2} \phi_{2}^{2}-\frac{1}{12} \phi_{2}^{4}+O\left(\phi^{5}\right)
$$

We will compute the residue of the S-matrix element $S_{22}$ at the pole corresponding to exchange of the particle $\phi_{1}$. We find it convenient to work with renormalized masses, changing however the overall mass scale so as to keep the mass of particle $\phi_{2}$ at its classical value $m_{2}^{2}=2$. This leads to an overall rescaling of the coupling constants since the mass scale enters in their definition. Additional $O\left(\beta^{4}\right)$ contributions arise from one-loop vertex corrections and wave-function renormalization.

The one-loop corrections to the $\langle 1,2,2\rangle$ vertex are represented by the diagrams in Fig. 8. a,b and give, respectively from the bubbles and the triangles,

$$
(a):-\frac{i \beta^{3}}{2 \sqrt{3}} \quad, \quad(b): i \beta^{3}\left(-\frac{1}{36 \sqrt{3}}+\frac{1}{6 \pi}\right)
$$

to be compared with the classical contribution $\langle 1|i \mathcal{L}| 2,2\rangle=2 i \beta$.

Additionally, there are contributions from wave-function renormalization, represented in Fig. 8.c, which give

$$
Z_{1}^{\frac{1}{2}} Z_{2}=1+\frac{\beta^{2}}{2}\left(\frac{5}{36 \sqrt{3}}-\frac{1}{2 \pi}\right)
$$


Figure 8: Feynman diagrams giving the one-loop correction to the $\langle 1,2,2\rangle$ vertex in the $a_{3}^{(2)}$ theory 
Figure 9: Feynman diagrams giving the one-loop correction to the $\langle 1,1,2\rangle$ vertex in the $c_{2}^{(1)}$ theory

to be multiplied into the classical coupling. Finally, there is an additional contribution to the coupling constant from the mass rescaling we performed in order to keep the mass of the particle $\phi_{2}$ at its classical value. This amounts to multiplying the classical coupling constant by

$$
1-\frac{\delta m_{2}^{2}}{m_{2}^{2}}=1+\frac{\beta^{2}}{12 \sqrt{3}}
$$

obtained from Ref. [7]. The total $O\left(\beta^{3}\right)$ value of the coupling is thus

$$
\langle 1,2,2\rangle=2 i \beta-2 i \beta^{3}\left(\frac{1}{9 \sqrt{3}}+\frac{1}{6 \pi}\right)
$$

and it leads to a contribution to the $S_{22}$ amplitude, in the vicinity of the $\phi_{1}$ particle pole

$$
\frac{1}{4 \widetilde{m}_{2}^{2} \operatorname{sh} \theta}\left(2 i \beta-2 i \beta^{3}\left(\frac{1}{9 \sqrt{3}}+\frac{1}{6 \pi}\right)\right)^{2} \frac{1}{s-\widetilde{m}_{1}^{2}}
$$

to be compared to order $\beta^{4}$ with the corresponding pole from the exact S-matrix

$$
S_{22}=\frac{(2)_{H}(H-2)_{H}}{(B)_{H}(2+B)_{H}(H-2-B)_{H}(H-B)_{H}}
$$

We find

$$
B=\frac{\beta^{2}}{2 \pi}-\frac{\beta^{4}}{8 \pi^{2}}
$$

which is indeed consistent with the expected form of $B$.

We have carried out the same calculation for the $c_{2}^{(1)}$ theory by studying the behavior of the $S_{12}$ amplitude in the vicinity of the $\phi_{1}$ pole. Here the classical masses are $m_{1}^{2}=4$ and $m_{2}^{2}=8$, and the relevant part of the interaction lagrangian is

$$
\mathcal{L}_{\text {int }}=2 \sqrt{2} \phi_{1}^{2} \phi_{2}-\frac{1}{3} \phi_{1}^{4}-2 \phi_{1}^{2} \phi_{2}^{2}-\frac{2}{3} \phi_{2}^{4}+O\left(\phi^{5}\right) .
$$

The one loop vertex corrections from Fig. 9.a,b are 


$$
(a):-\frac{3 i \beta^{3}}{\sqrt{2}} \quad, \quad(b): i \beta^{3}\left(\frac{1}{2 \sqrt{2}}+\frac{1}{\sqrt{2} \pi}\right)
$$

as compared to the tree level value $\langle 1,1|i \mathcal{L}| 2\rangle=4 \sqrt{2} i \beta$. The wave-function renormalization factors represented in Fig. 9 give

$$
Z_{1} Z_{2}^{\frac{1}{2}}=1+\beta^{2}\left(\frac{1}{16}-\frac{5}{16 \pi}\right)
$$

and from the shift in the mass scale one obtains an additional correction to the vertex

$$
1-\frac{\delta m_{2}^{2}}{m_{2}^{2}}=1+\frac{\beta^{2}}{16}
$$

leading to a total value of the coupling

$$
\langle 1,1,2\rangle=4 \sqrt{2} i \beta-i \beta^{3} \frac{3 \sqrt{2}}{4}\left(1+\frac{1}{\pi}\right)
$$

Comparison to the exact S-matrix $S_{12}=\{2\}_{H}\{H-2\}_{H}$ again checks the correct identification of $B$.

\section{References}

[1] A.B. Zamolodchikov, "Integrable Field Theory from Conformal Field Theory", Proceedings of the Taniguchi Symposium, Kyoto (1988).

[2] A.E. Arinshtein, V.A. Fateev and A.B. Zamolodchikov, Phys. Lett. 87B (1979) 389.

[3] H.W. Braden, E. Corrigan, P.E. Dorey and R. Sasaki, Phys. Lett. 227B (1989) 441; Nucl. Phys. B338 (1990) 689; Nucl. Phys. B356 (1991) 469.

[4] C. Destri and H.J. de Vega, Phys. Lett. 233B (1989) 336.

P. Christe and G. Mussardo, Nucl. Phys. B330 (1990) 465; Int. J. Mod. Phys. A5 (1990) 4581;

P.G.O. Freund, T.R. Klassen and E. Melzer, Phys. Lett. B229 (1989) 243.

[5] G.W. Delius, M.T. Grisaru, S. Penati and D. Zanon, Phys. Lett. 256B (1991) 164; Nucl. Phys. B359 (1991) 125.

[6] A.B. Zamolodchikov and Al.B. Zamolodchikov, Ann. Phys. (NY) 120 (1979) 253.

[7] M.T. Grisaru, S. Penati and D. Zanon, Phys. Lett. 253B (1991) 357.

[8] G.W. Delius, M.T. Grisaru and D. Zanon, CERN-TH.6301/91, to be published in the proceedings of "Strings and Symmetries 91", Stony Brook conference, May 1991. 
[9] G.W. Delius, M.T. Grisaru and D. Zanon, "Quantum conserved currents in affine Toda theories", CERN-TH.6336/91, in preparation.

[10] H.W. Braden and R. Sasaki, "Affine Toda Perturbation Theory", YITP/U-9140 or Edinburgh-/90-91/02.

[11] G.W. Delius, M.T. Grisaru and D. Zanon, CERN-TH.6333/91, Milano IFUM 409/TH, Phys. Lett. B (to be published).

[12] H.W. Braden and R. Sasaki, Phys. Lett. 255B (1991) 343; H.S. Cho, J.D. Kim, I.G. Koh and R. Sasaki, "Perturbative Verification of S-matrices in Affine Toda Field Theories", YITP/U-91-44 or KAIST-THP-91/701.

[13] M.A. Olshanetsky, Comm. Math. Phys. 88 (1983) 63.

[14] M.T. Grisaru, S. Penati and D. Zanon, Brandeis preprint BRX-TH-320, Milano IFUM-401-FT, Nucl. Phys. B (to be published).

[15] T.R. Klassen and E. Melzer, Nucl. Phys. B338 (1990) 485. 